\title{
A Survey of App Store Analysis for Software Engineering
}

\author{
William Martin, Federica Sarro, Yue Jia, Yuanyuan Zhang and Mark Harman
}

\begin{abstract}
App Store Analysis studies information about applications obtained from app stores. App stores provide a wealth of information derived from users that would not exist had the applications been distributed via previous software deployment methods. App Store Analysis combines this non-technical information with technical information to learn trends and behaviours within these forms of software repositories. Findings from App Store Analysis have a direct and actionable impact on the software teams that develop software for app stores, and have led to techniques for requirements engineering, release planning, software design, security and testing. This survey describes and compares the areas of research that have been explored thus far, drawing out common aspects, trends and directions future research should take to address open problems and challenges.
\end{abstract}

Index Terms-App Store, analysis, mining, API, feature, release planning, requirements engineering, reviews, security, ecosystem

\section{INTRODUCTION}

App stores are a recent phenomenon: Apple's App Store and Google Play were launched in 2008, and since then both have accumulated in excess of 1 million downloadable and rateable apps. Google announced that there were 1.4 billion activated Android devices in September 2015 [32]. Mobile app stores are also extremely lucrative: the set of online mobile app stores were projected to be worth a combined 25 billion USD in 2015 [152]. The success of app stores has coincided with the mass consumer adoption of smartphone devices. Smartphones existed prior to the launch of these stores, but it was not until 2008 that users could truly exploit their extra computing power and resulting versatility through downloadable apps. In-house and even commercial applications had been available before the launch of app stores, but app stores had some differences: availability, compatibility, ease of use, variety, and user-submitted content.

It is the user-submitted content that fundamentally distinguishes app stores from the ad-hoc commercially available applications that existed beforehand. As a result, software engineering researchers have access to large numbers of software applications together with customer feedback and commercial performance data, unavailable in previous software deployment mechanisms.

Furthermore, through readily available, downloadable toolkits, users can write their own applications to make use of a smart device's hardware. They can subsequently publish their software in the central app store for users to download (and possibly pay for). This publication process is subject to the store's in-house review and certification policies, but in general apps and app updates can be made available quickly (typically within hours/days).

In this paper we provide a survey of literature that performs "App Store Analysis for Software Engineering" between 2000 and November 27, 2015 ${ }^{1}$. Our contributions are as follows: i) We provide formal definitions of apps, stores, and technical and non-technical attributes, which are used

1. This paper is an updated version of an earlier technical report [157].
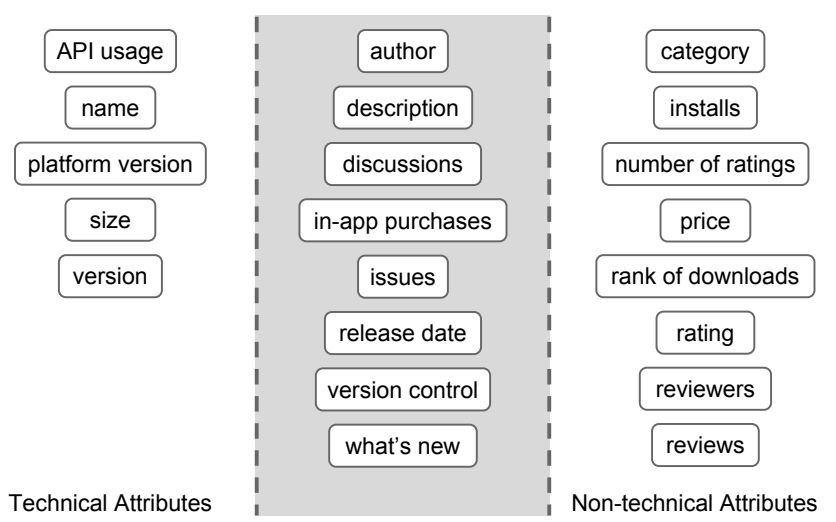

Fig. 1. Example attributes showing mined attributes that are strictly technical (left) or non-technical (right), and attributes that may be in either category (centre in box).

for App Store Analysis research. ii) We study the growth patterns of App Store Analysis literature both overall, and in each emergent subcategory. iii) We analyse the scale of app samples used, and discuss how this is likely to progress in the future. iv) We identify some of the key ideas published in App Store Analysis, in addition to common aspects, trends and future directions, to help readers to understand the progression of the field overall.

\subsection{Definitions}

The following definitions help to clarify key components of App Store Analysis literature. We used them to find all the relevant literature.

App: An item of software that anyone with a suitable platform can install without the need for technical expertise.

App Store: A collection of apps that provides, for each app, at least one non-technical attribute.

Technical attribute: An attribute that can be obtained solely from the software.

Non-technical attribute: An attribute that cannot be obtained solely from the software. 
Examples of attributes are shown in Figure 1, based on the data we collected in previous studies [92], [154], [202]. As our diagram shows, some attributes are distinctly technical or non-technical in a boolean sense, but others lie in a grey area, depending on the precise interpretation of what can be obtained from software alone. Those in the grey box cannot be considered technical in the strictest sense of the definition, because they are not guaranteed to be obtainable solely from the software in all cases. These attributes can be both nontechnical and technical, depending on how they are obtained. They are attributes that, in some cases, can be provided by the developer and not the app store, whilst attributes that are strictly non-technical may only be provided by an app store. For example, consider the 'author' attribute. In the case of Android software, the author can be obtained solely from the distributed apk file. However, in the case of a compiled c binary such as a simple "hello world" program, the author cannot be obtained directly from the binary file. The 'author' attribute therefore belongs in the grey area. We can obtain the size of the $\mathrm{C}$ binary, and so this attribute is technical; we cannot obtain the price from either of these example files, and so this is a non-technical attribute.

Our definition of App Store may seem simplistic. However, at the time of writing, app stores serve as more than just collections of apps, but enable more developers than ever to produce and distribute content, and enable a communication channel between users and developer via reviewing systems. Therefore, our definition is aimed at inclusivity. In only 7 years since the launch of the two biggest app stores, there are already over 180 papers devoted to their study, and each of these stores has well over 1 million apps each. As this rapid development has shown, the concept of apps and app stores is very likely to evolve over the coming years. It is our aim to encompass this evolution as best we can through the stated definitions, in the hope that future surveys will be able to build upon this work and the App Store Analysis literature to come.

\subsection{Overview}

This survey is structured as follows: Section 2 describes the process used to find the included literature; Section 3 breaks down the growth trends in non-technical research compared with technical-only research, and Section 4 breaks down the growth of scale of apps used; key ideas in each subfield of app store analysis are identified in Section 5.

We define the following App Store Analysis subfields, based on the literature gathered through the process explained in Section 2: "API Analysis", which is discussed in Section 6; "Feature Analysis", which is discussed in Section 7; "Release Engineering", which is discussed in Section 8; "Review Analysis", which is discussed in Section 9; "Security", which is discussed in Section 10; "Store Ecosystem", which is discussed in Section 11; and "Size and Effort Prediction", which is discussed in Section 12.

Closely related work is discussed in Section 13; guidelines and recommendations for future app store analysis authors are outlines in Section 14; we identify potential future directions in Section 15, and conclude our findings in Section 16.

\section{Literature SeARCH}

In this section, we describe the process used to find literature, including our scope, search terms and repositories and lessons learned for future app store analysis surveyors.

\subsection{Scope}

App Store Analysis literature encompasses studies that perform analysis on a collection of apps mined from an App Store. We are particularly interested in studies that combine technical with non-technical attributes, as these studies pioneer the new research opportunities presented by app stores. However, we also include studies that use app stores as software repositories, to validate their tools on a set of real world apps, or by using specific properties such as the malware verification process apps go through before being published in the major app stores.

Our survey is not a Systematic Literature Review (SLR). The area of App Store Analysis is still developing, but has not reached a level of maturity at which research questions can be chosen and asked of a well-defined body of literature. Our study aims to define, collect and curate the disparate literature, arguing and demonstrating that there does, indeed, exist a coherent area of research in the field that can be termed "App Store Analysis for Software Engineering". We hope that this will prove to be an enabling study for future SLRs in this area.

We apply the following inclusion criteria:

i) The paper is related to software engineering, and may have actionable consequences for software users, developers or maintainers.

ii) The paper is related to mobile app stores, concerning the use of collections of apps or non-technical data gathered from one or more app stores.

We apply the following exclusion criteria:

i) The paper focuses on mobile app development but does not extend to collections of apps nor to app stores.

ii) The paper uses an arbitrary collection of apps to test a tool, but it was not mined from an app store, and the study does not extend to app stores.

\subsection{Search Methodology}

In order to collect all relevant literature to date that meets the scope defined in Section 2.1, we perform a systematic search for the terms defined below, from each repository (also defined below). Unique papers are collected into a table, and a decision is made based on the inclusion criteria in three stages:

Title: We remove publications that are clearly irrelevant from the title.

Abstract: We inspect the abstract and remove publications which are clearly irrelevant according to the scope defined in Section 2.1.

Body: Results are read fully and a judgement is made on whether the paper a) meets the key requirements on what is defined as "app store analysis" in our scope, or b) is very relevant to the field and so should be included as "expanded literature", to put the main literature into context. Papers matching the requirements of a) or b) are included in this survey. 
A summary of the number of papers found through the search, as well as the number of papers accepted at each stage of validation, can be found in Table 1. All of the references for papers discussed in this survey are available in an online repository [201].

\subsubsection{Search Repositories}

We performed a search in each of the following repositories for papers to include in the study: Google Scholar, Scopus, JSTOR, ACM, IEEE and arXiv.

\subsubsection{Terms}

We searched for the following terms and phrases, to encompass the sub-fields of App Store Analysis that we identify: "App Store", mining, API, feature, release, requirements, reviews, security, and ecosystem. We performed searches for the following specific queries, where terms joined by an 'AND' must appear, and phrases in quotes must appear verbatim:

"app store analysis"

"app store analysis" AND mining

"app store analysis" AND mining AND API

"app store analysis" AND mining AND feature

We performed the following more general searches to ensure that no relevant literature was missed from the survey:

"app store" AND analysis AND API

"app store" AND analysis AND API AND mine

"app store" AND analysis AND feature AND mine

"app store analysis" AND mining AND requirements

"app store analysis" AND mining AND release

"app store analysis" AND mining AND reviews

"app store analysis" AND mining AND security

"app store analysis" AND mining AND ecosystem

We mitigate the threat of missing papers by conducting searches for "app store analysis" AND "mining" and also each of the names of each of the major subfields of App Store Analysis literature. Since, by our definition, app store analysis research uses collections of apps, this should encompass much of the field. We also performed snowballing, which further helps to mitigate the threat of potentially missing papers. However, the threat of missing papers is a threat to the validity of any survey, including this one.

\subsection{Snowballing}

In addition to the repository searches specified in Section 2.2, we also perform snowballing [244] on many of the included studies. To do this we inspect the studies cited by the study, and the publications that subsequently cited the study, using Google Scholar and ACM. By performing this process in addition to repository keyword searching, we reduce the risk that relevant literature is omitted from this survey.

\subsection{Search Results}

Search results can be found in Table 1 .

We set the time window to start with the year 2000, yet the earliest reported study is 2010 . This is likely because the App Stores that propelled mobile app usage to become widely adopted were launched in 2008. Yet, it is interesting that studies incorporating technical with non-technical app store information did not emerge until two years later. Papers were collected until November 27, 2015.

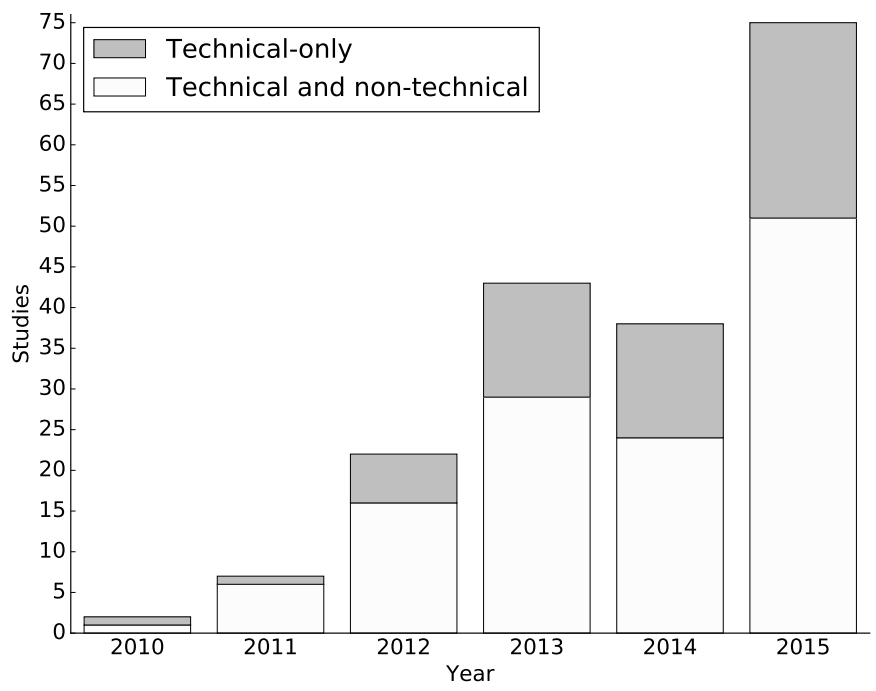

Fig. 2. Histogram showing number of research papers incorporating non-technical information and technical-only research papers showing the period from 2010 to November 27, 2015.

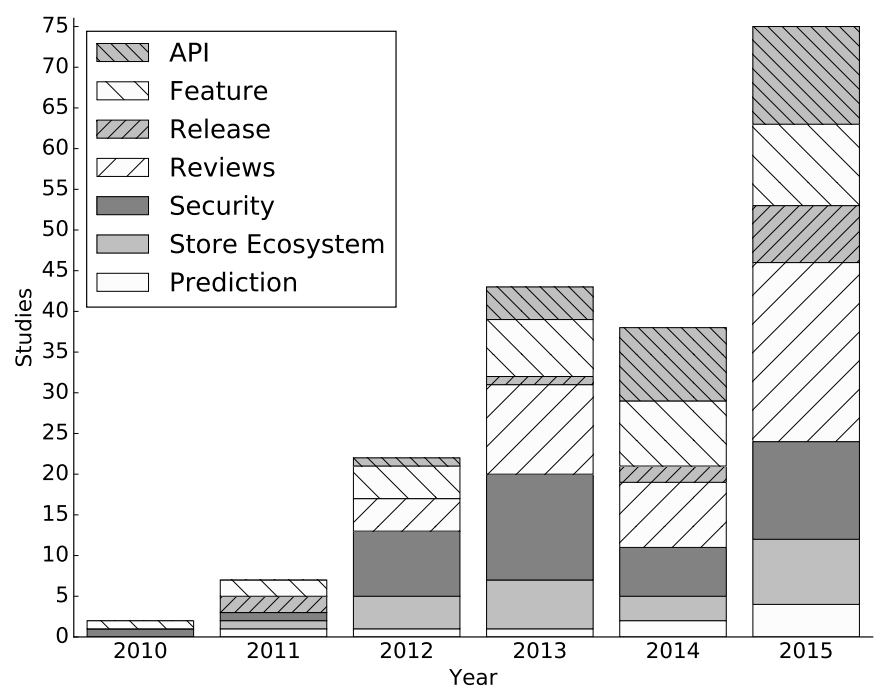

Fig. 3. Histogram of sub-field trends showing the period from 2010 to November 27, 2015.

An overlap was found between search queries performed, and thus the total number of discovered papers through search queries was fewer than suggested by the sum of the bottom rows in Table 1 . Many papers were discovered through snowballing, which do not appear in the table.

We present a summary of the included literature in Tables 3 to 9. Histograms depicting the growth of publications studied on App Store Analysis for software engineering can be found in Figures 2 to 4, which show the split between technical-only and technical and non-technical research, the split between different subfields identified as subsections in this survey, and the split between scale of studies in terms of the number of apps used, respectively. A breakdown of these studies in each sub-field that we identify is also presented in Figure 5.

\subsection{Lessons Learned}

As can be seen from Table 1, for some queries, there were large drops in the number of papers upon inspection of their 
TABLE 1

Search query results indicating the number of hits each query generates, the number of these that were available to be inspected, the number of titles and subsequent abstracts and paper bodies that were accepted as valid. The top boxes indicate more specific queries run in multiple paper repositories, and the lower boxes indicate the more general queries run only in Google Scholar. In the case of Google Scholar, only the top 1,000 results were accessible to inspect at the time of search.

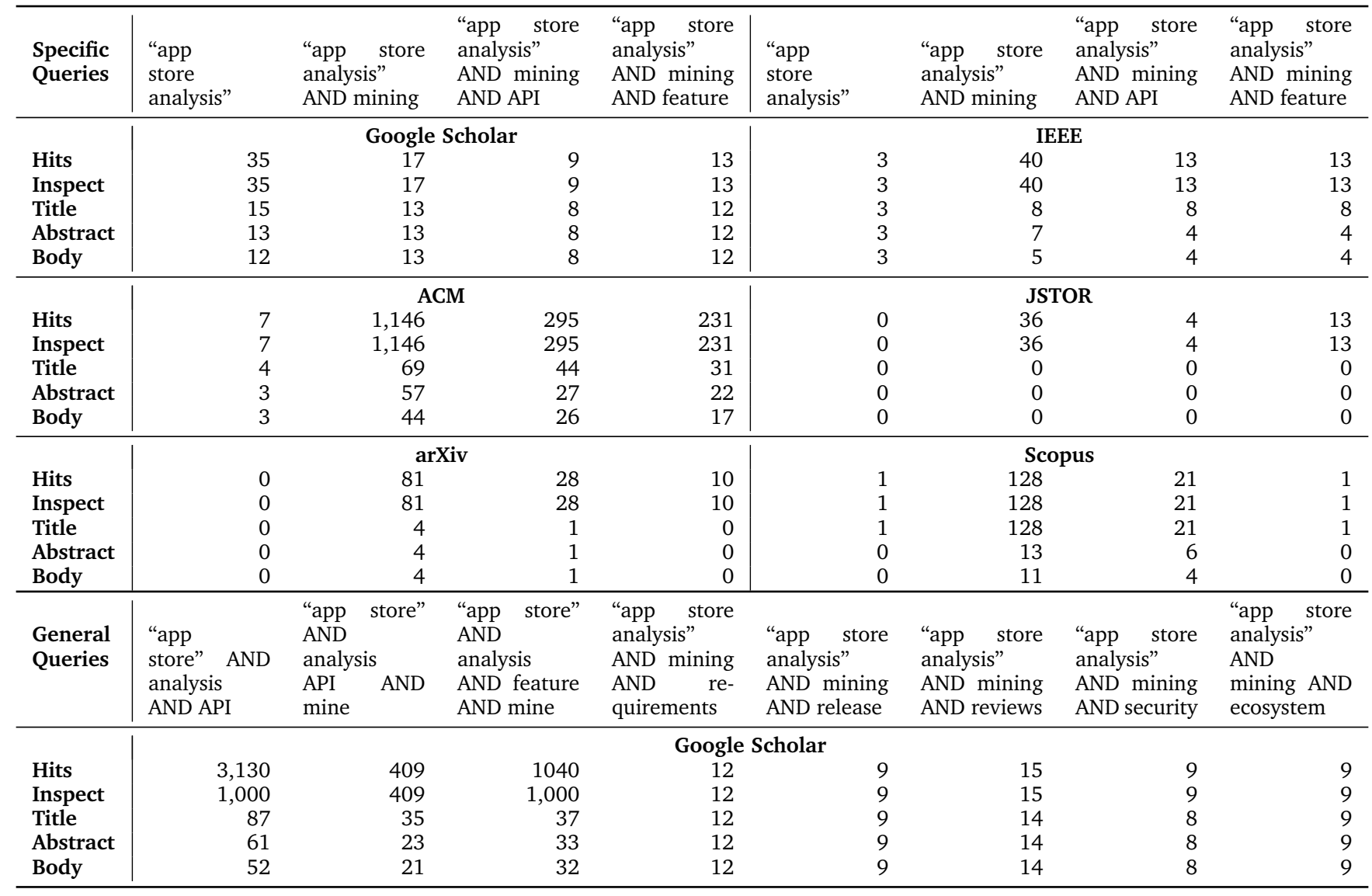

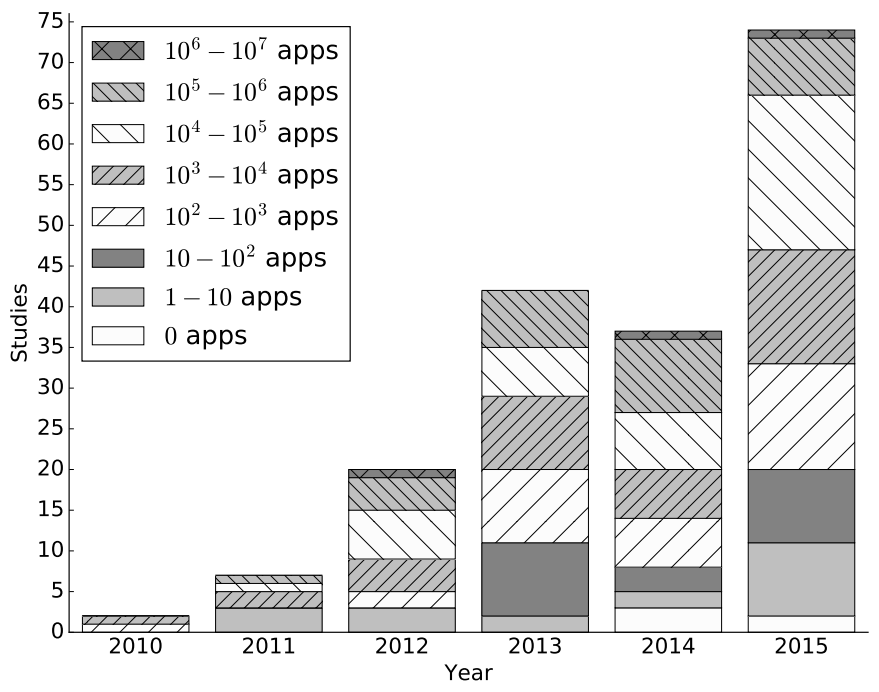

Fig. 4. Histogram showing number of research papers grouped into app quantity ranges each year, showing the period from 2010 to November 27, 2015. Each histogram depicts a range such as $10^{2}$. $10^{3}$ apps, which means that the studies included used between $10^{2}$ and $10^{3}$ apps.

title or abstract, when performing the more general searches on Google Scholar: searches for "app store" with many of the combinatoral words resulted in several thousand papers

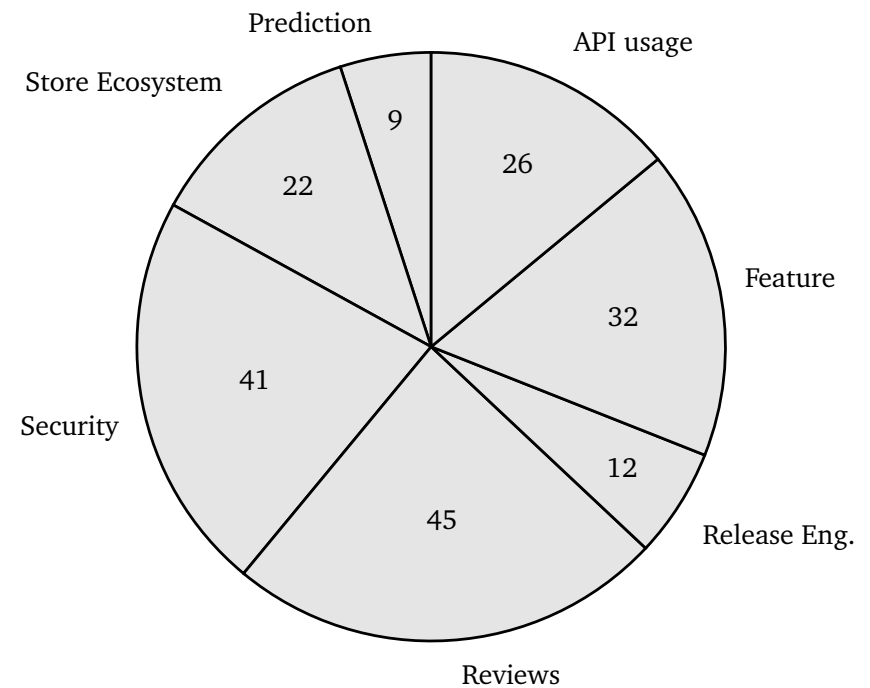

Fig. 5. Pie chart showing overall sub-field distribution showing the period from 2010 to November 27, 2015.

which may have mentioned "app store" only once. We found that searching for "app store analysis" as a phrase narrowed the results down a lot, but did miss some relevant papers.

Searches that included "mining" as a keyword did encompass much of app store analysis research due to the 
TABLE 2

Number of research papers studying each app quantity range from 2010 to November 27, 2015.

\begin{tabular}{lr|lr}
\hline No. Apps Range & Papers & No. Apps Range & Papers \\
\hline 0 & 5 & {$\left[10^{3}, 10^{4}\right)$} & 36 \\
{$[1,10)$} & 19 & {$\left[10^{4}, 10^{5}\right)$} & 39 \\
{$\left[10,10^{2}\right)$} & 21 & {$\left[10^{5}, 10^{6}\right)$} & 28 \\
{$\left[10^{2}, 10^{3}\right)$} & 31 & $\geq 10^{6}$ & 3 \\
\hline
\end{tabular}

focus on collections of apps that meets our app store definition. However, we found that the snowballing technique was crucial in our literature search, because paper discovery through many of the paper repositories we used could not be replied upon to find all relevant papers; in a growing field terms of reference are not fully stabilized. We therefore encourage future surveyors to visit the App Store Analysis paper repository [201], which can assist in the discovery of app store analysis literature.

\section{Non-Technical Research}

While software engineering deals primarily with code, it is not confined to deal with strictly technical sources of information. We can combine data from multiple (technical and nontechnical) sources, and app stores provide a wealth of such information. There are 127 of 187 (68\%) papers included in this study that incorporate non-technical information mined from app stores in order to either infer technical attributes (such as features), or to extract useful information such as bug reports and feature requests from users.

The histogram in Figure 2 shows that the number of studies incorporating non-technical information is growing year-on-year. We can see from Figure 2 that even including the boom in technical-only research, there is growth year-onyear (with the exception of 2014). Using linear regression, we are able to fit the growth trend with high accuracy $\left(R^{2}=0.9067, p=0.003373\right)$, which indicates that we can draw a straight line and predict (with $90 \%$ accuracy) the publications for a given year.

\section{Scale of Studies}

In order to discuss the number of apps that are studied by research papers, we first need to define a set of ranges. We assign the papers studied to app quantity ranges in ascending powers of 10, according to the number of apps that they consider. The ranges that we assign, and the number of research papers that study them, are shown in Table 2.

The median number of apps used in the considered literature is 1,679 , and the mean is 44,807 . This result shows that half of the papers study fewer than 2,000 apps, but the other half study a quantity of apps several orders of magnitude larger. This is reflected in Figure 4, where the range $\left[10^{4}, 10^{5}\right)$ is shown to grow and in 2015 represents almost half of the app usage literature.

The histogram for the studies using between $10^{4}$ and $10^{5}$ apps shows growth from 2011 to 2015, and this result is reflected in the histogram for studies using between $10^{5}$ and $10^{6}$ apps as well, up to 2014. It is important to note that we did not have complete data for 2015 , so this result is subject to change. Studies using smaller scales of apps show an
TABLE 3

Chronological summary of API-related App Store Analysis

literature showing the authors, publication year, publication venue, and the number of apps used in the study.

\begin{tabular}{llr}
\hline Authors [Ref], Year & Venue & No. apps \\
\hline Ruiz et al. [195], 2012 & ICPC & 4,323 \\
Linares-Vásquez et al. [138], 2013 & FSE & 7,097 \\
Shirazi et al. [196], 2013 & EICS & 400 \\
Minelli and Lanza [163], 2013 & ICSM & 20 \\
Minelli and Lanza [164], 2013 & CSMR & 20 \\
Ruiz et al. [193], 2014 & IEEE Soft. & 236,245 \\
Hao et al. [91], 2014 & MobiSys & 3,600 \\
Dering and McDaniel [57], 2014 & MILCOM & 450,000 \\
Linares-Vásquez et al. [140], 2014 & MSR & 24,379 \\
Ruiz et al. [192], 2014 & IEEE Soft. & 208,601 \\
Linares-Vásquez [137], 2014 & ICSE comp. & 0 \\
Viennot et al. [226], 2014 & SIGMETRICS & $1,107,476$ \\
Bartel et al. [18], 2014 & IEEE Soft. Eng. & 1,421 \\
Zhang et al. [250], 2014 & WiSec & 10,311 \\
Borges and Valente [30], 2015 & PeerJ C. S. & 396 \\
Bavota et al. [21], 2015 & IEEE Soft. Eng. & 5,848 \\
Kim et al. [121], 2015 & ASE & 350 \\
Khalid et al. [114], 2015 & IEEE Soft. & 10,000 \\
Watanabe et al. [242], 2015 & SOUPS & 200,000 \\
Zhou et al. [254], 2015 & WiSec & 36,561 \\
Wan et al. [236], 2015 & ICST & 398 \\
Wang et al. [237], 2015 & ISSTA & 105,299 \\
Syer et al. [214], 2015 & Soft. Qual. & 5 \\
Azad [15], 2015 & Masters thesis & 950 \\
Wang et al. [238], 2015 & UbiComp & 7,923 \\
Seneviratne et al. [204], 2015 & WiSec & 4,114 \\
\hline & Mean & 93,298 \\
& Median & 5,086 \\
\hline
\end{tabular}

uncertain change in frequency, indicating that most studies in the future are likely to continue using over $10^{4}$ apps. We anticipate larger studies in the future, based on the growth of App Store Analysis literature, the increasing quantity of apps studied, and of course the growing app stores themselves.

\section{Key IDEAs Timeline}

A timeline depicting the key ideas is shown in Figure 6. This highlights the launch of major app stores studied, as well as the first studies in each subsection. We include studies into the timeline that have advanced the field of App Store Analysis in some way, or introduced influential ideas into their respective subsection.

\section{API ANALYSIS}

Papers that extract the API usage from app APKs or source code, and combine this information with non-technical data are discussed in this section, and are summarised in Table 3. All API analysis literature studied apps from the Android platform only. This may be due to the availability of tools which can be used to decompile the apps and extract their API calls, which are freely available and can be applied to downloaded app binaries. It is perhaps surprising that such analyses have not also been performed on the Apple platform, iOS, since the store was launched in 2008. This might be because iOS binaries are only available for the intended platforms, and cannot be downloaded to, or used from a desktop computer without an Apple Developer account, which is not free. Even with such an account, app binaries or source code would be needed, and neither are freely available due to a) copyright 


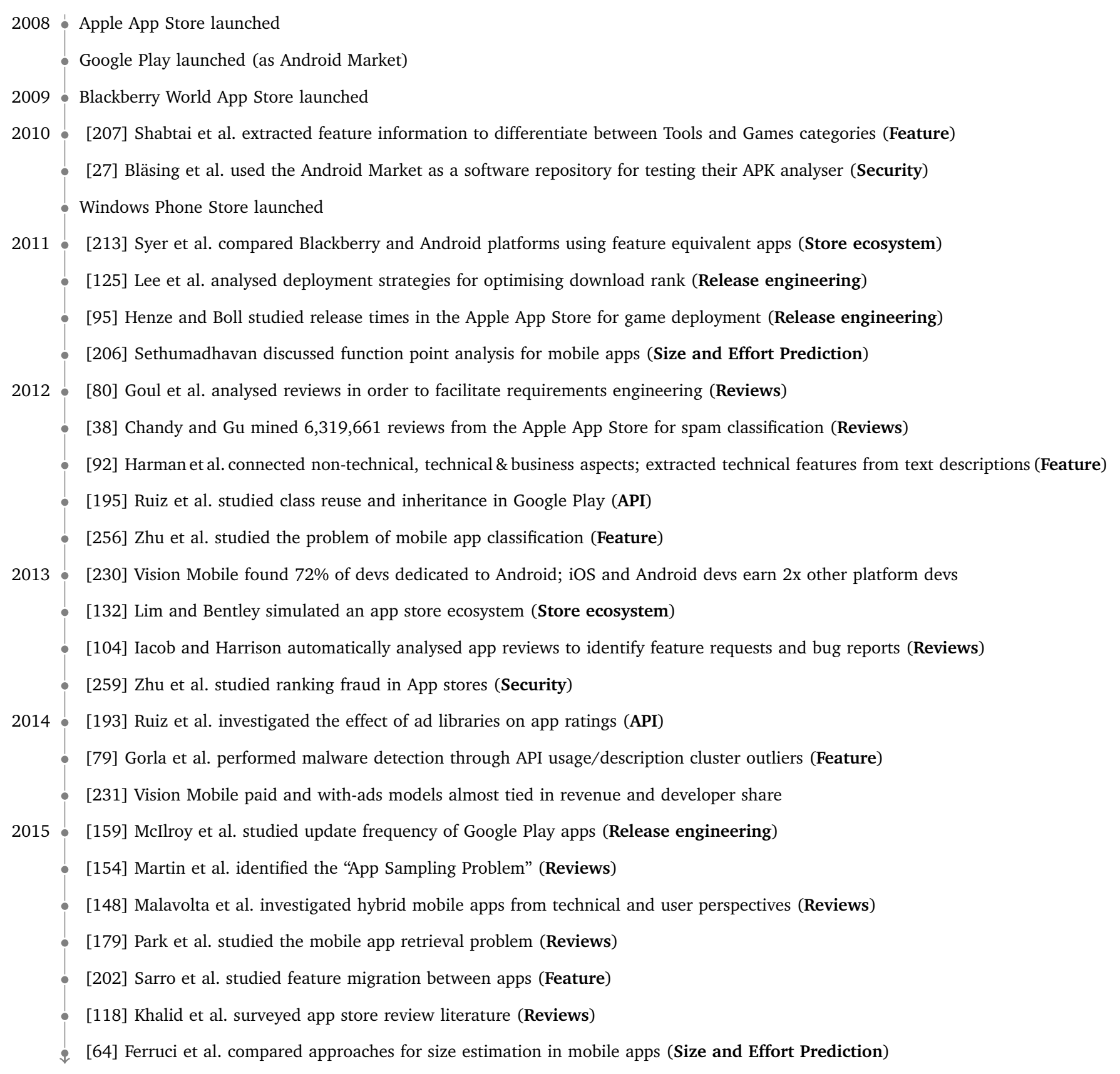

Fig. 6. Key ideas timeline for App Store Analysis literature. The primary area of study is suffixed in parentheses.

on binaries and b) many iOS apps being paid-for apps. Due to these difficulties, it is uncertain whether it will be possible for future studies to extract API information from iOS apps; in fact, it may become harder since the move (in iOS9) to developer-submitted LLVM IR (Intermediate Representation) binaries, which are then compiled for specific platforms by Apple.

API analysis literature can be decomposed into "API Usage", "Class Reuse and Inheritance", "Faults" and "Permissions and Security". There is some overlap between the latter subsection and Section 10 . Nevertheless, the literature discussed in this Section is collected together and discussed here because it directly analyses API usage. Several papers included in this section relate to energy usage [139], [236], although much of this field of research relates only indirectly to app stores. For those who wish to learn more on the subject, we point the reader to the recent paper by Hindle [96].

All API analysis literature has, hitherto, studied apps from the Android platform. There is large range in the number of apps considered, from 0 apps to over $1,000,000$.

\subsection{API Usage}

Borges and Valente [30] used association rule mining to infer API usage patterns, using a dataset of 396 open source Android apps. For their study, the authors extended 
APIMiner [167] to mine usage patterns and instrument API documents with extracted usage patterns. They reported a study over 17 months, during which instrumented Android documentation was made publicly available, and received approximately 78,000 visits. Shirazi et al. [196] extracted the API usage with regards to user interface (UI) elements and layout, and compared statistics between the 21 different categories of the Google Play store that existed in 2012.

Wan et al. [236] explored energy hotspots in apps by transforming their UIs and producing a ranked list of UI components by energy consumption. The authors tested their approach on 398 apps mined from Google Play. Azad [15] studied apps mined from Google Play and F-droid, and produced tools to inspect API usage and suggest similar APIs based on Stackoverflow discussions, score the similarity of apps, identify the degree to which apps have copied the source code of open source projects, and detect license violations. Tian et al. [219] extracted API information and evaluated apps in terms of code complexity, API dependency, API quality, as well as a number of other factors, in order to train features to distinguish high from low rated apps.

API usage can be extracted from Android APK files, making analysis on the Android platform relatively straightforward. The extracted information has been used to analyse energy usage, detect malware, analyse graphical elements and to detect license violations.

\subsection{Class Reuse and Inheritance}

In 2012 Ruiz et al. [195] studied class reuse and inheritance in 4,323 Android apps mined from 5 categories in Google Play. Of these, 217 apps were found to contain exactly the same set of classes as another app in the same category. The study was later extended to 208,601 apps by Ruiz et al. [192] in 2014. More evidence of substantial code reuse was found, and the authors concluded that app developers benefit from increased productivity but risk dependence on the quality of the code they reuse.

In 2013 Minelli and Lanza presented a visual analytics web tool for studying repositories of apps [163], [164]. The tool analyses snapshots of apps throughout their version history, using an interactive graphical user interface. Following their subsequent study on 20 free and open source Android apps, the authors found that 3rd party API code is often (incorrectly) committed along with the app code, instead of including the corresponding 3rd party jar files. Excluding 3rd party code, most apps were found to have comparatively small code-bases. Additionally the authors found little use of inheritance in Android apps, and much duplication. Viennot et al. [226] introduced the P layDrone Google Play crawler, which they used to store daily data on 1.1M apps and decompile $880 \mathrm{k}$ free apps. The authors found that native libraries are heavily used in popular apps, and that approximately a quarter of free apps are duplicates of other apps. They found that paid apps account for just $0.05 \%$ of downloads, and the top $10 \%$ of most popular apps account for $96 \%$ of total downloads as of June 23, 2013.

Linares-Vásquez et al. [140] decompiled and analysed 24,379 APKs from Google Play and found that the $82 \%$ of de- tected clones replicate 3rd party libraries. Zhang et al. [250] proposed ViewDroid, an app plagiarism detection system that uses view transition graphs as "birthmarks" to capture app behaviour, in order to detect clones in the presence of code obfuscation. Apps mined from Google Play were used as a false negative set. In a related study, Kim et al. [121] scan API invocations to identify plagiarised applications, in a more sophisticated approach than similarity detectors that scan code, as it handles code obfuscation. Wang et al. [237] proposed WuKong, a two-phase Android clone detection system that first filters third-party libraries to increase detection speed. The authors tested the system on 105,299 Android apps and found zero false positives.

Code reuse is common in the Google Play store, but inheritance use is comparatively rare. Most apps are found to have small code bases, often replicating third party code instead of including compiled jar files. Clone and plagiarism detection tools are a widely discussed topic in the Class Reuse and Inheritance literature.

\subsection{Faults}

Linares-Vásquez et al. analysed the effect of fault and changeprone core Google APIs on app ratings [138]. This is an important study as it combines technical API information with non-technical information in the form of average user reviews, in order to assess the impact that API usage can have. Fault and change prone APIs were found to be used more frequently by poorly-rated apps. Conversely, popular apps used APIs that were found to be less susceptible to faults and changes. The paper presents an analysis of 7,097 randomly selected free apps with $>10$ reviews. Changes and faults were measured as the number of API changes and bug fixes, respectively, to the particular associated core libraries.

Building on the work by Linares-Vásquez et al. [138], Linares-Vásquez also presented an approach for a recommendation system for Android app developers [137], to help them to prepare for platform updates and avoid breaking changes and introducing bugs. The authors extended their API analysis work to identify APIs that have a high energy usage [139], but this study did not combine non-technical app store information.

Bavota et al. [21] investigated how the number of changes and faults present in APIs used affected apps' ratings. Their results showed an inverse correlation between the popularity of apps and the number of faults and changes in APIs they used. That is, low rated apps were found to use APIs that are more fault- and change-prone than highly rated apps. Bavota et al. surveyed 45 Android developers who confirmed this relationship from anecdotal experience. These studies combined technical (API usage) with non-technical (user ratings) information to highlight best practice for API usage in Android development.

Syer et al. [214] studied the effect of platform independence on source code quality, finding that the more defect prone source files also depend more heavily on the platform. The authors therefore suggest prioritising platformdependent source files for unit testing, as a quality assurance strategy. In 2015, Khalid et al. [114] performed static analysis 
on 10,000 free Google Play apps, and found that 3 categories of $\mathrm{F}$ indBugs warnings occur more frequently in lower rated apps. The categories 'bad practice', 'internationalisation' and 'performance' had more warnings in lower-rated apps, suggesting that these areas are the ones developers should focus on to achieve better rating performance.

Fault and change-prone APIs have been used more frequently by poorly rated apps. Fault prone apps often depend more heavily on the platform than non fault prone apps.

\subsection{Permissions and Security}

In 2013 Peiravian and Xingquan [180] used API calls and permissions data to train their malware classifier, which they trained and validated on 1,260 malware samples and 1,250 benign samples, using cross-validation. Hao et al. [91] studied the insertion of UI handlers into app code. They published the PUMA tools which makes UI automation programmable, and enables researchers to analyse correctness properties of apps. They tested the tool on a set of 3,600 apps downloaded from Google Play. Dering and McDaniel [57] downloaded a set of 700,000 app binaries from 450,000 free apps on Google Play and analysed library and permission usage. They found a strong correlation between the number of libraries used and the number of permissions requested by the apps, leading to the conclusion that libraries tend to have specific use cases that require additional permissions from the user. This finding presents a security concern: is each library doing what it is supposed to, and does it need this permission? In conjunction with the finding by Book et al. [29], this suggests that library usage is a significant security concern, since libraries often make use of existing permission privileges, and also increase the number of permissions requested.

Ruiz et al. studied the effect of advertisement libraries on app ratings [193]. They combined non-technical rating information with the extracted technical information showing advertisement library usage to perform the study. Advertisement libraries query their host server at regular intervals to fetch advertisements for display, and this interval determines the "advertisement fill rate". Multiple libraries are often used to obtain higher fill rates in order to increase revenue. From a sample of 236,245 apps, the authors found no evidence of a correlation between rating and the number of advertisement libraries. However, certain APIs were found to have low median ratings from apps that used them. The authors state that this is due to intrusive behaviours, such as recording entered passwords.

Gorla et al. [79] trained a one-class support vector machine [149] on API usage information in order to identify outliers in trained clusters for security purposes. Bartel et al. [18] showed that off-the-shelf static analysis is insufficient for permission-protected API methods, and investigated alternatives, which they tested on 1,421 apps downloaded from two Android markets. Watanabe et al. [242] found, from analysing the description and API usage of 200,000 Android apps, that there is disparity between their descriptions and requested permissions. This is due to a combination of factors: unnecessary permissions requested by app building frameworks, or developers that use similar manifests for multiple app projects; secondary functionality that is not mentioned in descriptions; and the use of 3rd party libraries. In a related study, Zhou et al. [254] mined a set of 36,561 Android apps, and proposed the tool CredMiner which is focused on decompilation and program slicing. They identified over 400 apps that leaked developer user-names and passwords, required for the program to execute normally.

Wang et al. [238] decompiled 7,923 apps from Google Play and mined features from the decompiled code and variable names. They trained a machine learning classifier on labelled instances of the apps using location and contact information, in order to identify the way in which sensitive information is used. Seneviratne et al. [204] studied 275 free and 234 paid Android apps, and found that paid apps collect personal information, in the same way as free apps do. $60 \%$ of the paid apps collected personal information, compared to $85 \%$ in free apps. The authors subsequently showed that $20 \%$ of 3,605 collected Android apps were connected to more than three trackers.

There is a strong correlation between libraries used and permissions requested. Advertisement libraries sometimes have intrusive behaviours such as recording entered passwords. The treatment of personal data is a topic of interest in Permissions and Security API analysis.

\subsection{Future Work}

The biggest available avenue for future API analysis literature is to consider alternative platforms: all studies thus far have extracted API usage from Android apps. It remains to be seem what effect the move to intermediate representation will have on potential API analysis in the Apple App Store, but it may hinder efforts. The Windows Phone platform is relatively recent, and we may start to see API analysis studies utilising this platform; the Google Play store launched in 2008 (as Android Market), but it was not until 2012 that App Store Analysis literature studied API usage in the store.

The scale of API analysis studies is large, but future work may seek to study how usage varies over long time periods. Literature has looked at how API usage differs between apps of varying popularity or rating, but there is potential to look at differences between categories.

\section{Feature Analysis}

Papers that extract feature information from either technical or non-technical sources of information are discussed in this subsection, and are summarised in Table 4. We can observe that these research papers study a wide range of platforms: Android, iOS, Nokia Widsets, Blackberry and Windows Phone. In addition, the publications investigate a large number of apps: the minimum is 3 and the maximum is 600,000 . 
TABLE 4

Chronological summary of feature-related App Store Analysis literature showing the authors, publication year, store used: $g$ signifies Google Play or other Android stores, a signifies Apple App Store, $n$ signifies the Nokia (or Widsets) platform, b signifies Blackberry, $s$ signifies Samsung (Android) and w signifies Windows Phone; publication venue, and the number of apps used in the study.

\begin{tabular}{lllr}
\hline Authors [Ref], Year & Store & Venue & No. apps \\
\hline Shabtai et al. [207], 2010 & g & CIS & 2,285 \\
Chen and Liu [40], 2011 & a & iConference & 102,337 \\
Coulton \& Bamford [49], '11 & $\mathrm{n}$ & MobileHCI & 3 \\
Harman et al. [92], 2012 & $\mathrm{b}$ & MSR & 32,108 \\
Sanz et al. [197], 2012 & $\mathrm{g}$ & CCNC & 820 \\
Teufl et al. [218], 2012 & $\mathrm{g}$ & MobiSec & 130,211 \\
Zhu et al. [256], 2012 & $\mathrm{n}$ & CIKM & 680 \\
Mokarizadeh et al. [166],'13 & $\mathrm{g}$ & WEBIST & 21,065 \\
Teufl et al. [217], 2013 & $\mathrm{g}$ & Sec. \& Com. Netw. & 443 \\
Lulu and Kuflik [22], 2013 & $\mathrm{g}$ & IUI & 120 \\
Bhattacharya et al. [25],'13 & $\mathrm{g}$ & CSMR & 24 \\
Yin et al. [249], 2013 & $\mathrm{a}$ & WSDM & 5,661 \\
Lin et al. [135], 2013 & $\mathrm{a}$ & SIGIR & 7,116 \\
Ihm et al. [107], 2013 & $\mathrm{g}$ & CGC & 10 \\
Kim et al. [122], 2014 & $\mathrm{a}$ & Service Business & 100,830 \\
Finkelstein et al. [65], 2014 & $\mathrm{b}$ & Tech. report & 42,092 \\
Yang et al. [248], 2014 & $\mathrm{g}$ & Tech report & 26,703 \\
Zhu et al. [257], 2014 & $\mathrm{n}$ & TMC & 680 \\
Zhu et al. [260], 2014 & $\mathrm{g}$ & KDD & 170,753 \\
Jiang et al. [109], 2014 & $\mathrm{g}$ & INTERNETWARE & 150 \\
Zhu et al. [255], 2014 & a & IEEE Cybernetics & 15,045 \\
Gorla et al. [79], 2014 & $\mathrm{g}$ & ICSE & 32,136 \\
Vakulenko et al. [222], 2014 & $\mathrm{a}$ & ICIS & 600,000 \\
Lin et al. [136], 2014 & $\mathrm{a}$ & SIGIR & 6,524 \\
Sarro et al. [202], 2015 & b,s & RE & 54,983 \\
Berardi et al. [24], 2015 & a,g & SAC & 5,993 \\
Svedic [212], 2015 & $\mathrm{a}$ & PhD thesis & 66 \\
Seneviratne et al. [205],'15 & $\mathrm{g}$ & WWW & 232,906 \\
Tong et al. [220], 2015 & g,w & JCST & 10,000 \\
Wang et al. [238], 2015 & $\mathrm{g}$ & UbiComp & 7,923 \\
He et al. [93], 2015 & $\mathrm{g}$ & Big Data & 122,875 \\
Tian et al. [219], 2015 & $\mathrm{g}$ & ICSME & 1,492 \\
Nayebi and Ruhe [172],'15 & $\mathrm{g}$ & PeerJ C.S. & 241 \\
Lulu and Kuflik [23], '15 & $\mathrm{g}$ & MOB INF SYST & 6,633 \\
Al-Subaihin et al. [4], '16 & $\mathrm{bg}$ & ESEM & 17,877 \\
\hline & & Mean & 51,203 \\
& & Median & 6,875 \\
\hline
\end{tabular}

Features have been extracted from app descriptions, API usage, manifest files, decompiled source strings, categories and permissions.

Papers in this section show that it is possible to extract feature information from sources other than source code or requirements lists. Additionally, many different methods are used for extraction and categorisation of features, including natural language processing, topic modelling and clustering. The work shows that analysis of app collections can be augmented with meaningful technically-oriented information, mined from freely-available app store pages.

Feature Analysis literature is broken down into "Classification", "Clustering", "Lifecycles", "Recommendation", "Success" and "Verification". This section has an overlap with Section 10, in the cases where features are used to help detect anomalies or verify app functionality.

\subsection{Classification}

Shabtai et al. [207] extracted feature information from the manifest, XML files, API calls and methods used from a set of 2,285 Google Play apps. They trained a classifier on the features to differentiate between Tools and Games categories, as a proof of concept that malware detectors could be trained in the same way. In 2012 Sanz et al. [197] trained machine learning classifiers to predict app categories, using extracted features. The features used for prediction were strings extracted from the decompiled app code, requested permissions, rating, number of ratings and app size. They tested the approach on 820 apps and found a peak AUC (area under ROC curve) of 0.93 using the Bayesian TAN classifier [67].

Zhu et al. [256], [257] studied the problem of mobile app classification in the Nokia Store. The authors mined 680 apps, and experimented by classifying apps using data from web search and from device logs from users of the apps. Their approach outperformed other classification techniques, and enabled them to automatically classify a given app onto a predefined category of Apple's App Store taxonomy. In 2015 Berardi et al. [24] built on this work, by constructing a classifier using features mined from app descriptions, categories, names, ratings and file sizes. They trained the classifier using a support vector machine for each of 50 classes, and used the BM25 weighting scheme [190] on the features. Users manually classified 5,993 apps mined from Apple App Store and Google Play, to act as the training (cross validation) set for the classifier.

Jinh et al. [110] used the features: numbers of app installs, number of reviews, category and rating score, in conjunction with features based on information flow, for their machine learning classifier for rating app security risk. Wang et al. [238] extracted features from decompiled Java code, from their collection of 7,923 apps mined from Google Play. They used the extracted features to train classifiers for predicting how 'location' and 'contact' information is used, with $85 \%$ and $94 \%$ accuracy, respectively.

Features have been extracted for use with classifiers, in order to differentiate categories, rate app security and to predict how sensitive information is used.

\subsection{Clustering}

Teufl et al. [218] mined 130,211 apps from Google Play and performed clustering on both app descriptions and requested permissions, as part of their activation patterns malware detection approach. They later extended this work [217] to propose a first-step malware detection method using links between description terms and security permissions to identify suspicious outliers. In 2013 Mokarizadeh et al. [166] performed clustering on 21,065 apps, mined from Google Play, after applying topic modelling on app descriptions. They found that the resultant clusters were very different from the apps' assigned categories, and apps in the same category often had dissimilar description topic distributions. Mokarizadeh et al. also performed correlation analysis and found that users downloaded free apps more frequently, and 
that downloads correlated with the number of ratings an app had received.

Lulu and Kuflik [22] performed clustering on 120 apps mined from Google Play, comparing description-based with category-based clustering. They found that descriptions provided good clustering features, and presented the method as the basis of an app recommendation system. The authors later built on this work [23], by extracting features from 6,633 app descriptions and enriching them with information mined from the web, found by searching for the app name. They used the enriched features to provide an installed-app recall interface, supported by functionality-based categorisation. The interface was validated by performing a user study with 40 participants, who were able to find apps faster and found the categorisation more intuitive, when compared with a reference "smart launcher" interface [74].

Kim et al. [122] mined 100,830 apps from Apple App Store, and extracted feature keywords from their descriptions using natural language processing. They clustered apps using the extracted features, and re-categorised them using the resulting clusters. Al-Subaihin et al. [4] mined 17,877 apps from Google Play and Blackberry World app store. The authors clustered the apps using the similarity between features extracted from their descriptions. They scored the quality of the resulting clusters, and found them to be of higher quality than the existing categorisation of the mined apps. The authors conducted a human assessment of app similarity within clusters of varying granularity, and found a strong correlation between the similarity score of their technique and the human assessment.

Descriptions are often used for clustering apps based on their functionality. Clustering has been used to assign categories and to identify outliers. The clusteringassigned categories tend to differ from the store-assigned categories, and have also been shown to outperform them in feature classification quality.

\subsection{Lifecycles}

Sarro et al. [202] proposed a theoretical characterisation of feature lifecycles in app stores, to help app developers to identify trends and to find undiscovered requirements. In order to investigate app feature migratory and nonmigratory behaviours in current app stores, they mined features from app descriptions using the techniques in the earlier work [92], and used the proposed theory to empirically analyse the migratory and non-migratory behaviours of 4,053 non-free features from Samsung and Blackberry stores. The results revealed that features generally migrated to a category with similar characteristics. However, there were also a few features that migrated to apparently non-related categories. The early identification of these features may allow developers to find undiscovered requirements. The authors also found that approximately one third of features were intransitive (they neither migrate nor do they die out over the period studied), and such features exhibited significantly different behaviours with regard to important properties, such as their price. Being aware of the intransitive features in a given category may support developers in identifying crucial ('must-have') requirements for their apps.

Features can migrate between apps and between categories. Intransitive features that do not migrate have been found to exhibit significantly different properties than migratory features.

\subsection{Recommendation}

Yin et al. [249] proposed the Actual Tempting (AT) model to perform app recommendation for users. The model incorporates latent tempting parameters. Take for example two apps, "a" and "b". The AT model incorporates the number of users who own app "a" and subsequently download app "b", and the number who do not download "b" after owning "a". The model also uses feature overlap information, measured by performing topic modelling on app descriptions and computing the topic overlap between each pair. The authors found that the AT approach outperformed collaborative filtering and case-based reasoning in their initial experiments.

Lin et al. [135] used topic modelling on the Twitter messages of users that follow an app's Twitter feed, in order to generate latent groups related to the app. The groups were then used as part of a recommendation system, in order to help remove the problem of cold start in app recommendation based on other metadata. The system was tested on 7,116 apps mined from Apple App Store, and the authors found that it outperformed recommendation using app descriptions. In 2014 Lin et al. [136] used topic modelling on app descriptions in order to produce a recommendation system. The model was semi-supervised and incorporated app version information using different weights corresponding to update types: so that newer app versions could be recommended when they add a certain feature to the description. Resultant topics were weighted based on their category in the app store to provide a recommendation. The model was trained on 6,524 apps mined from the Apple App Store.

Zhu et al. [255] mined the daily top 300 free and top 300 paid apps from Apple App Store charts from February 2, 2010 to September 17, 2012, collecting information on 15,045 apps in total. They used popularity information to construct a Popularity-based Hidden Markov Model (PHMM), to encode trend and other latent factors. The authors stated that this can be used in a variety of ways, including app recommendation, review spam detection, and demonstrated its usefulness in ranking fraud detection. Zhu et al. [260] built an app recommendation system using a combination of technical information (device permissions requested) and non-technical information (app popularity). They tested the system on 170,753 apps mined from Google Play to show its scalability. However, the system received no human-based evaluation of its recommendations.

Valulenko et al. [222] performed topic modelling on a set of 600,000 app descriptions mined from the Apple App Store. They used the resultant topics to suggest categories, and to improve and augment existing categorisation approaches used in app stores. He et al. [93] trained a system for targeting users for advertising, with a dataset containing 
app install data on a per-user basis, consisting of 122,875 apps from the Huawei App Store. The authors reported a higher click rate than targeting approaches existing at the time of writing. Nayebi and Ruhe [172] extracted feature information from 241 Google Play apps, and used crowdsourcing to assign user value to each of the features. The authors used the approach for service portfolio planning [2].

A variety of models have been trained on app feature data, incorporating hidden 'latent' factors, that are subsequently used to recommend apps to users, target users for advertising, and to suggest categories.

\subsection{Success}

In 2011, Coulton and Bamford [49] conducted a case study on games created for the WidSets platform, an earlier app store for Nokia phones (including non-smartphones). Their findings are transferable to modern app stores: high download numbers were required in order to gain active users, and popular features such as chat were able to increase the popularity and the proportion of active users. Chen and Liu [40] collected 102,337 apps from Apple App Store, and observed no correlation between download rank and rating, from a sample of the top 200 most popular apps.

Harman et al. [92] introduced app store mining as an MSR (Mining Software Repositories) problem. They mined app information and performed correlation analysis on price, downloads, and rating. Correlation analysis was performed in both app and feature space, where features were extracted using natural language processing techniques from app descriptions, and results showed that under most conditions there is a strong correlation between rating and downloads (popularity). The proposed approach can be applied to different app stores by modifying the data extraction and parsing phases to accommodate the different app store structure and data representations. The authors later extended this work [65], finding that free apps have higher ratings than non-free apps, with a medium effect size. They also carried out a developer survey on the extracted features, who found them meaningful, and were able to successfully detect the extracted features over randomly generated features.

Bhattacharya et al. [25] presented an empirical study of 24 open source Android apps from multiple categories, with the aim of defining metrics of bug report quality and developer involvement. The authors showed how the bug-fix process is affected by differences in bug lifecycles. Security bug reports were found to be of higher quality, but the associated bugs are fixed more slowly. The scale of the study was large as all apps had more than 1,000 ratings, 100,000 downloads and 200 bug reports. The authors found that bug report quality correlates with description length but not app rating.

Ihm et al. [107] conducted a study on 10 popular apps in the Google Play store, analysing the correlations between app downloads in the store and external metrics. The authors found a strong positive correlation between the number of downloads in the store and the number of registered users on the app's respective websites, and a strong correlation between the number of downloads and the app website (inverse) download rank. Jiang et al. [109] conducted a user survey on 50 app descriptions in order to identify the attributes most important to the quality of a description. A support vector machine was trained on the resultant attributes and tested on a sample of 100 descriptions, finding an accuracy of 0.62 . The findings showed that quick overviews were the most effective form of app description, and the study contains further heuristics on good description styles.

In a longitudinal study on 60 paid iOS apps, Svedic [212] found that ratings and reviews can impact sales ranks. The study found that higher, more stable ratings lead to users associating the app with high quality, and the app sales increased as a result. Tian et al. [219] studied 1,492 high and low rated apps from Google Play, and identified the features which most accurately differentiate apps with high rating from those with low rating. The authors used technical features, such as code complexity and API usage, with nontechnical information such as the category and the number of images displayed on the app store page. The most important features for differentiating high from low rated apps were the size of the app and the number of images on store page. The target SDK version was also an influential feature, which suggests that high rated apps were updated more frequently and used more modern features of the Android operating system.

Ratings and reviews have been found to correlate with sales and download ranks. Features mined from app descriptions can be used as a basis for correlation analysis, and have been found meaningful from a developer survey.

\subsection{Verification}

Yang et al. [248] introduced the APPIC framework, which extracts main theme tag words from Android description and permission files. It does this using LDA and Partially Labelled Dirichlet Allocation (PLDA), for the purpose of identifying misleading app descriptions. It uses an app's permissions file to establish whether its description makes claims consistent with its functionality, and whether it resides in an appropriate category. The method was tested on 207,865 apps from Google Play, and was manually evaluated on a subset of 1,000 apps. The authors found that their method achieved (average) $88.1 \%$ category accuracy, and $76.5 \%$ permissions accuracy.

Watanabe et al. [242] found that apps often contain secondary functionality that is not mentioned in their descriptions. In a study of 232,906 apps, Seneviratne et al. [205] trained a machine learning classifier on app features in order to detect spam apps. The features used for the classifier were numeric statistics about an app's description. The authors labelled apps that were removed from the store and establishing potential reasons for removal. Apps likely to have been removed due to being spam (the majority of those removed) were then used to train a boosting classifier in order to identify potential spam.

Tong et al. [220] proposed the App Generative Model (AGM) topic model, for extracting semantically coher- 
TABLE 5

Chronological summary of release engineering-related App Store Analysis literature showing the authors, publication year, store used: g signifies Google Play or other Android stores, a signifies Apple App Store and w signifies Windows Phone; the type of literature, and the number of apps used in the study.

\begin{tabular}{lllr}
\hline Authors [Ref], Year & Store & Venue & No. apps \\
\hline Lee and Raghu [125], 2011 & a & AMCIS & 3,168 \\
Henze and Boll [95], 2011 & a & MobileHCI & 24,647 \\
Datta and Kajanan [54], 2013 & a & CloudCom-Asia & 3,535 \\
Lee and Ragu [126], 2014 & a & JMIS & 7,579 \\
Ruiz et al. [194], 2014 & $\mathrm{g}$ & IEEE Soft. & 120,981 \\
Guerrouj et al. [84], 2015 & $\mathrm{g}$ & SANER & 154 \\
Comino et al. [45], 2015 & a,g & Tech report & 1,000 \\
McIlroy et al. [159], 2015 & $\mathrm{g}$ & ESE & 10,713 \\
Gui et al. [85], 2015 & $\mathrm{g}$ & ICSE & 21 \\
Carbunar and Potharaju [33],'15 & $\mathrm{g}$ & ASONAM & 160,000 \\
Alharbi and Yeh [6], 2015 & $\mathrm{g}$ & MobileHCI & 24,436 \\
Martin et al. [153], [155], 2016 & g,w & ICSE comp. & 1,033 \\
Martin et al. [156], 2016 & $\mathrm{g}$ & FSE & 38,858 \\
\hline & & Mean & 29,772 \\
& & Median & 5,557 \\
\hline
\end{tabular}

ent app features from descriptions, using term co-occurrence statistics. The AGM model resulted in lower perplexity (a topic model fitness function that measures the log-likelihood of generating a held-out test set), than the most commonly used model, LDA. However, the model precision was evaluated only against TF.IDF, and not LDA or similar topic models such as the weighted topic model [162]. Nevertheless, the study shows the importance of accurate feature discovery and representation, and can help lead to future studies using extracted features.

Features have been used in a classifier for spam detection, and to validate whether an app makes correct claims about its functionality.

\subsection{Future Work}

There is potential for future work in tracking feature migration in alternative app stores: thus far the literature has studied apps mined from Blackberry and Samsung stores. Additionally, future work may seek to investigate the migration of features between different app stores or platforms.

Features have been used to classify and cluster apps, as well as recommend similar apps or categories. However, future work may apply recommendation in a different direction: it could be very useful for developers to receive recommendations on features they might implement, based on similar apps, highly desirable intransitive features, or other methods.

\section{Release Engineering}

This section discusses papers that focus on app releases or release strategies, which are summarised in Table 5. We can see from Table 5 that there were two papers published in 2011 that tackle this issue, one in 2013, and then a recent influx of five prior to November 27, 2015. Release studies typically require time series data, in order that the changes made to apps in their releases can be recorded. The scale of the past studies in this section is relatively small, ranging from 21 to 160,000; this scale is not surprising, given the difficulty of mining longitudinal data for a large number of data points.

Release Engineering literature has featured Apple and Google platforms but not yet Blackberry, Samsung or Windows. The scale in studies has been small, most likely due to the difficulty in obtaining time series data.

Release Engineering literature is broken down into "Content", "Success" and "Strategy" subsections.

\subsection{Content}

The 2014 study by Ruiz et al. investigated the updates made to update advertisement libraries [194]. They found that over 12 months, almost half of the 5,937 apps with multiple updates had an advertisement library update. Approximately $14 \%$ of advertisement updates contained no changes to the app's code, indicating the effort involved in keeping advertisement libraries updated. Gui et al. found, from 21 apps in Google Play with frequent releases, that $23 \%$ of their releases contained ad-related changes [85].

The findings of Guerrouj et al. [84] indicate that high code churn in releases correlates with lower ratings. Alharbi and Yeh [6] tracked the design patterns used by 24,436 Android apps over a period of 18 months. They found that depreciated patterns were sometimes adopted after they are depreciated, and that new pattern adoption rates were low. By tracking the app descriptions, they found that app developers sometimes updated the app descriptions to reflect changes in their applied design patterns. The authors believe that this shows that descriptions are used as a communication channel between developers and users. The authors report on apps that start and stop using certain design patterns. An interesting future research direction might be to record the migration of these "design features" using the app feature migration terminology of Sarro et al. [202].

Up to half of app updates over a 12 month period are advertisement library updates, which have been found to contain no other changes in $14 \%$ of cases. High code churn has been found to correlate with lower ratings.

\subsection{Success}

Moller et al. [170] studied the installation behaviour of users with recently updated apps, in a security related study. Lee and Raghu [126] studied the factors that affect an app's likelihood of staying in the top (most popular) charts in the Apple App Store. They found that free apps are more likely to 'survive' in the top charts, and that frequent feature updates are the most important factor in ensuring their survival, along with releasing in smaller categories. The authors also found that high volumes of positive reviews improve an app's likelihood of survival.

Carbunar and Potharaju [33] conducted a longitudinal study on 160,000 Google Play apps mined daily over a 6 
month time period in 2012. They found that at most 50\% of apps were updated in each category, and that there is an issue of "stale apps" affecting aggregated statistics on large populations. The authors also found that a few developers dominated the total download counts, that productive developers did not have many popular apps, and that there was no correlation between price and downloads.

Martin et al. [153], [155] conducted a longitudinal study on 1,033 apps mined from Google Play and Windows Phone Store over a 12 month time period. The authors used causal inference to identify the releases with most impact on ratings and downloads. They found that release text discussing features and not bug fixes may have led to more significant releases, and releases that improved rating. Martin et al. [156] later extended this work on a sample of 38,858 apps from Google Play, using their tool, CIRA. They found that paid apps that had significant positive effects on success were more expensive. The authors also contacted the developers of significant releases, finding that $78 \%$ agreed with the causal assessment and $33 \%$ would consider changing their release strategy based on findings from the study.

Positive reviews and frequent feature updates can help to keep free apps in the "most popular" charts. Causal inference has been used to identify releases with high impact on ratings and downloads.

\subsection{Strategy}

Lee et al. [125] published the earliest work that meets our definition of "app store analysis" in 2011 by incorporating technical with non-technical information for analysis of apps. The authors mined app information from the top 300 iOS apps in all 21 categories free and paid, mining at least 3,168 apps. They analysed developer diversification through publishing apps in multiple categories and in both free and paid sections, and found a positive relationship between download rank and app portfolio diversification. The study incorporated technical (download rank) with non-technical information (category, price) in order to identify actionable findings for app developers.

Henze and Boll [95] analysed release times and user activity in the Apple App Store, and concluded that Sunday evening was the best time for deploying games. Their study also found that version updates were an effective strategy for raising an app's rank in the store. Datta and Kajanan [54] studied review counts from the Apple App Store, and found that apps received more reviews after deploying updates on Thursdays.

In 2014 Lin et al. [136] incorporated version information in their app recommendation system, in order to ensure that apps were recommended if they added new features to updated versions. Comino et al. [45] studied the top 1,000 apps in Apple App Store and Google Play. They found that for iTunes, increased numbers of app releases were more likely when the app was performing badly, and that releases could boost downloads. Neither finding held true for Google Play, however.

McIlroy et al. [159] studied update frequencies in the Google Play store, after mining data about 10,713 mobile apps. They found that only $1 \%$ of the studied apps received more than one update per week, and only $14 \%$ were updated in a two-week period. The authors also found that rating was not affected by update frequency. Nayebi and Ruhe [172] combined app features with values gained from crowdsourcing as an approach to app service portfolio planning.

App updates have been found to be more likely when an app is performing badly, and releases can boost downloads in the Apple App Store. Multiple studies suggest that day of release is a factor in the immediate success of app releases.

\subsection{Future Work}

Due to the 2015 spike in release engineering studies, we expect the trend to continue and contribute to the growing numbers of App Store Analysis literature. As can be seen in Table 5, the stores studied are split almost equally into Apple and Google, but there is potential future work involving release studies on Blackberry or Windows Phone Store.

Several studied in this section have looked at developer strategy, and release behaviours that associate with positive results. A potential for future work could be individualised recommendation of strategy, which could be particularly useful for app developers who wish to break into higher app store ranks.

\section{Review Analysis}

Literature discussed in this section concerns the study of app reviews; a summary of discussed literature can be found in Table 6. We can see from Table 6 that the majority of studies focused on the Google Play store, with a minority focusing on Apple App Store, and 1 paper studying Blackberry store. Review-centred literature was first published in 2012, and subsequently has gained significant and increasing interest and activity: we can see from Figure 3 that there are greater numbers of requirements/reviews literature each year. We hypothesise that this is due to the tenure of the stores, and the progression of the field.

Review Analysis literature mostly studies Apple and Google stores, inviting future comparison with Windows and other store reviews.

Literature in this section is broken down into "Classification", "Content", "Requirements Engineering", "Sentiment", "Summarisation" and "Surveys and Methodological Aspects of App Store Analysis". Many early works have focused on the content of reviews in 2012-2013, before advancing to sentiment in 2013-2014, and requirements and summarisation in 2015.

\subsection{Classification}

Chandy and $\mathrm{Gu}[38]$ mined 6,319,661 reviews from 3,090 apps in the Apple App Store. After manually labelling a subset of the mined reviews as spam or not spam, the authors trained both a supervised decision tree and unsupervised latent class analysis to identify spam reviews. The unsupervised 
TABLE 6

Chronological summary of reviews-related App Store Analysis

literature showing the authors, publication year, store used: $g$ signifies

Google Play or other Android stores, a signifies Apple App store, b

signifies Blackberry; the type of literature, and the number of apps used in the study.

\begin{tabular}{|c|c|c|c|}
\hline Authors [Ref], Year & Store & Venue & No. apps \\
\hline Hoon et al. [101], 2012 & $\mathrm{a}$ & OzCHI & 17,330 \\
\hline Vasa et al. [224], 2012 & a & $\mathrm{OzCHI}$ & 17,330 \\
\hline Chandy and Gu [38], 2012 & a & WebQuality & 3,090 \\
\hline Goul et al. [80], 2012 & a & HICSS & 9 \\
\hline Ha et al. [89], 2013 & $\mathrm{~g}$ & CCNC & 59 \\
\hline Oh et al. [174], 2013 & $\mathrm{~g}$ & $\mathrm{CHI}$ & 24,000 \\
\hline Hoon et al. [100], 2013 & a & Tech report & 17,330 \\
\hline Iacob and Harrison [104], 2013 & $\mathrm{~g}$ & MSR & 270 \\
\hline Galvis Carreño et al. [69], 2013 & $\mathrm{~g}$ & ICSE & 3 \\
\hline Khalid [115], 2013 & a & ICSE & 20 \\
\hline Fu et al. [68], 2013 & $\mathrm{~g}$ & $\mathrm{KDD}$ & 171,493 \\
\hline Chen et al. [42], 2013 & $\mathrm{a}, \mathrm{g}$ & WWW & 5,059 \\
\hline Pagano and Maalej [175], 2013 & $\mathrm{a}$ & $\mathrm{RE}$ & 1,100 \\
\hline Hoon et al. [99], 2013 & $\mathrm{a}$ & OzCHI & 25 \\
\hline Iacob et al. [106], 2013 & $\mathrm{~g}$ & BCS-HCI & 161 \\
\hline Iacob et al. [105], 2014 & $\mathrm{~g}$ & MobiCASE & 270 \\
\hline Khalid [117], 2014 & a & IEEE Soft. & 20 \\
\hline Chen et al. [41], 2014 & $\mathrm{~g}$ & ICSE & 4 \\
\hline Cen et al. [36], 2014 & $\mathrm{~g}$ & PIR & 6,938 \\
\hline Guzman and Maalej [88], 2014 & $a, g$ & $\mathrm{RE}$ & 7 \\
\hline Khalid et al. [116], 2014 & $\mathrm{~g}$ & FSE & 99 \\
\hline Wano and Iio [241], 2014 & a & NBIS & 500 \\
\hline Erić et al. [61], 2014 & a & QIP & 968 \\
\hline Khalid et al. [118], 2015 & $\mathrm{~g}$ & IJITCS & 0 \\
\hline Gao et al. [70], 2015 & g & SOSE & 4 \\
\hline McIlroy et al. [160], 2015 & $a, g$ & ESE & 12,000 \\
\hline Cen et al. [35], 2015 & $\mathrm{~g}$ & SIAM & 12,783 \\
\hline Vu et al. [234], 2015 & $\mathrm{~g}$ & ASE & 3 \\
\hline Vu et al. [233], 2015 & $\mathrm{~g}$ & CoRR & 95 \\
\hline Malavolta et al. [147], 2015 & $\mathrm{~g}$ & MS & 11,917 \\
\hline Malavolta et al. [148], 2015 & g & MOBILESoft & 11,917 \\
\hline Park et al. [179], 2015 & $\mathrm{~g}$ & SIGIR & 43,041 \\
\hline Panichella et al. [178], 2015 & $\mathrm{a}, \mathrm{g}$ & ICSME & 7 \\
\hline Palomba et al. [176], 2015 & $\mathrm{~g}$ & ICSME & 100 \\
\hline Moran et al. [168], 2015 & $\mathrm{~g}$ & FSE & 14 \\
\hline Gomez et al. [78], 2015 & $\mathrm{~g}$ & MOBILESoft & 46,644 \\
\hline Martin et al. [154], 2015 & $\mathrm{~b}$ & MSR & 15,095 \\
\hline Maalej and Nabil [146], 2015 & $a, g$ & $\mathrm{RE}$ & 1,140 \\
\hline Pérez [228], 2015 & $\mathrm{~g}$ & Masters thesis & 4 \\
\hline Khalid et al. [119], 2015 & - & IJIEEB & 0 \\
\hline Gu and Kim [83], 2015 & $\mathrm{~g}$ & ASE & 17 \\
\hline Guzman et al. [86], 2015 & $a, g$ & ESEM & 7 \\
\hline Guzman et al. [87], 2015 & $\mathrm{a}, \mathrm{g}$ & ASE & 7 \\
\hline McIlroy et al. [161], 2015 & $\mathrm{~g}$ & IEEE Soft. & 10,713 \\
\hline \multirow[t]{3}{*}{ Liang et al. [128], 2015} & a & IJEC & 139 \\
\hline & & Mean & 9,594 \\
\hline & & Median & 161 \\
\hline
\end{tabular}

method achieved higher accuracy, and took into account factors such as average rating of a user, and number of apps rated.

Chen et al. [42] compared the maturity ratings of 1,464 equivalent apps between the Apple App Store and Google Play, and taking the Apple store ratings as the accurate ratings, the authors found that $9.7 \%$ of the Android apps were underrated and $18.1 \%$ were overrated. The authors also studied a sample of 729,128 reviews from 5,059 Google Play game apps, and trained a classifier on the sets of app descriptions and user reviews, and iOS maturity ratings, to automatically verify app maturity ratings. Ha et al. [89] manually examined 556 reviews mined from 59 Google Play apps, in order to classify them into topics and sub-topics based on content. They found that most information in reviews concerned the quality of the app, and not security or privacy concerns.

Cen et al. [36] devised an approach to identify the Comments with Security / Privacy Issues (CSPI) from a set of mined Google Play app reviews. The authors later built upon this work, using reviews in order to rank the security risk of apps, by detecting security labels in a crowd-sourced approach [35]. Using AndroGuard [8] scores as a ground truth, the authors found that their tool outperformed other metrics for ranking app security risk, half of which incorporated user reviews and half of which relied on declared permissions.

Gomez et al. [78] used an unsupervised machine learning approach in order to identify apps that may contain errors, using 1,402,717 reviews mined from 46,644 apps. The authors used the error information in addition to permissions used by the apps, in order to construct a ranked recommender system to analyse app permissions, for app store moderators. Guzman et al. [87] developed an ensemble of machine learning classifiers in order to classify user reviews. They tested this system on 4,550 reviews mined from 7 apps in the Google and Apple app stores, and achieved a precision of 0.74 and recall of 0.59 on a manually labelled set of 1,820 reviews.

Reviews have been classified for spam, maturity ratings, and privacy and security risks. Research in 2015 has also used reviews to help detect erroneous apps.

\subsection{Content}

Hoon et al. [101] and Vasa et al. [224] collected a dataset containing 8.7 million reviews from the Apple App Store and analysed the reviews and vocabulary used. In 2013 Hoon et al. analysed 8 million reviews from Apple App Store [100]. They found that the majority of mobile app reviews were short in length, and that rating and category both influenced the length of reviews. The majority of studied apps received under 50 reviews in their first year. Half of the apps analysed decreased in the user assessment of quality, denoted by rating over time. The authors suggested that user expectations were changing rapidly towards apps, and that developers must keep up with demand to remain competitive.

Iacob et al. [106] studied how the price and rating of an app influence the type and amount of user feedback that it receives through reviews. The authors selected 3,279 reviews for the study, from which they identified 9 classes of feedback: positive, negative, comparative, price related, request for requirements, issue reporting, usability, customer support, versioning. From the selected apps, there was a roughly equal split of positive type reviews with feature/issue type reviews, with very few other types such as negative or price related.

Khalid et al. [116] studied the devices used to submit app reviews, in order to determine the optimal devices for testing. Palomba et al. [176] studied the Google Play reviews from 100 open source Android apps, and linked the reviews to code changes. They found that a mean of $49 \%$ of review requests were implemented in new releases, and that the apps with changes more directly implementing the content of user reviews improved their ratings with new releases. In order to bridge the gap between software attributes and user 
reviews, Hoon et al. [98] developed an ontology of words used to describe software quality attributes in app reviews.

McIlroy et al. [161] studied responses to reviews from 10,713 Google Play apps, finding that most developers do not respond to reviews. However, in the cases where a response occurred, $38.7 \%$ of users were found to subsequently change their ratings, resulting in a median increase in individual user ratings of $20 \%$. A summary of mobile app user feedback classification can be found in the study by Maalej et al. [235].

Review content literature has investigated the vocabulary and ontology of reviews, the factors affecting feedback, and devices most used for review submission.

\subsection{Requirements Engineering}

Oh et al. [174] developed a review digest system, that they tested on 1,711,556 reviews mined from 24,000 Google Play apps. They automatically categorised reviews into bug reports, functional requests and non-functional requests, and produced a digest featuring the most informative reviews in each category.

Iacob and Harrison [104] presented an automated system (MARA) for extracting and analysing app reviews in order to identify feature requests. The system is particularly useful because it offers a simple and intuitive approach to identifying requests. 161 apps and 3,279 reviews were used for manually training linguistic rules. 136,998 reviews were used for the evaluation, which found that $23.3 \%$ of reviews contained feature requests.

As an extension to the MARA system they had previously introduced [106], Iacob et al. [105] introduced a set of linguistic rules for identifying feature requests and bug reports in order to help facilitate app development. Wano and Iio [241] analysed the textual content of 856 reviews from 500 apps in the Japanese App Store, and found that the review styles differed between apps in different categories. In a large scale study, Erić et al. [61] studied the star ratings of 48 million reviews mined from 968 popular free and paid Apple apps. They found that the reviews were mostly positive, and that there were significant differences in the distributions between categories, and also between free and paid sections. Free apps had more reviews but a lower mean rating, and higher standard deviation. Due to the higher numbers of reviews for free apps, which might give an app credibility, the authors argued that in-app purchasing revenue models were a good way to make money for developers, especially if used as a 'teaser' for a paid version.

Park et al. [179] developed AppLDA, a topic model designed for use on app descriptions and user reviews, that discards review-only topics. This enables developers to inspect the reviews that discuss features present in the app descriptions. The authors tested the system on 1,385,607 reviews mined from 43,041 apps. Panichella et al. [178] presented a system for automatically classifying user reviews based on a predetermined taxonomy, in order to support software maintenance and requirements evolution. They verified the system on a manually labelled truth set of 1,421 sentences extracted from reviews, and achieved a precision of 0.85 and recall of 0.85 , when training the system on language structure, content and sentiment features. Maalej and Nabil [146] produced a classification method identifying bug reports and feature requests from user reviews. The authors found that upwards of $70 \%$ precision and $80 \%$ recall could be obtained using multiple binary classifiers, as an alternative to a single multiclass classifier. They also found that the commonly used NLP techniques, stopword removal and lemmatisation, could negatively affect the performance of this classification task.

Moran et al. [168] proposed the FUSION system, that performs static and dynamic analysis on Android apps, in order to help users complete bug reports. The system focuses on the steps to reproduce a bug, using dynamic analysis to walk through Android system events. Khalid et al. [119] argued that app store reviews can be used for "crowdsourcing" [150]. They argued that users are inadvertently performing crowdsourcing when they review apps, solving the following problems: requests for potential features, suggestions for developer action, recommendations for other users, and issue reporting.

The requirements engineering review literature has used reviews to extract bug reports and feature requests, in addition to prioritising critical reviews.

\subsection{Sentiment}

The works discussed in this subsection have incorporated sentiment in their study of reviews. Sentiment describes a user's views or opinions, typically as positive or negative in this content, and is extracted from reviews using 'positive' sentiment words such as 'good,great,love', or 'negative' sentiment words such as 'bad,hate,terrible'.

In 2012 Goul et al. [80] published the earliest work to study online app store reviews. The authors performed sentiment analysis on 5,000 Apple App Store reviews in order to facilitate requirements engineering. Galvis Carreño and Winbladh [69] extracted user requirements from comments using the ASUM model [111], a sentiment-aware topic model. Initial results showed that the method aided requirements summarisation with significantly less effort than manual identification, but did not return all possible requirements. Hoon et al. [99] gathered a set of 29,182 short reviews of up to 5 words from the top 25 Health \& Fitness apps in the Apple App Store. They analysed the reviews and found that they are mostly made up of sentiment words, which matched the star rating of the review closely.

Khalid [115], [117] manually categorised 6,390 negative reviews from a sample of 20 free iOS apps, and reported the most frequent causes of complaints. The apps had over 250,000 reviews combined, and therefore 6,390 reviews is a statistically representative sample at the $95 \%$ confidence level. The authors carried out a manual analysis of the 6,390 reviews, finding that $11 \%$ of samples concerned complaints about a recent update. Users were most dissatisfied by issues relating to invasion of privacy and unethical behaviour, while hidden cost was the second most negatively perceived complaint. Pagano and Maalej [175] gathered a sample of 1.1 million reviews from the Apple App Store in order to provide an empirical summary of user reviewing behaviour. 
They found that most feedback was provided after releases, that positive feedback was often associated with highly downloaded apps, and that negative feedback was often associated with less downloaded apps and often did not contain user experience or contextual information.

In 2014 Chen et al. [41] produced a system for extracting the most informative reviews, placing weight on negative sentiment reviews. Guzman and Maalej [88] studied user sentiments towards app features from a multi-store sample, and studied the differences between user sentiments in Google Play from the Apple App Store. Guzman et al. [86] developed a tool called DIVERSE, that extracts key reviews specific to a queried feature. DIVERSE groups together reviews with similar sentiments about the same feature in order to condense the information. The authors tested their tool al. [128] performed MultiFacet Sentiment Analysis (MFSA) on user reviews mined from 139 apps on the Apple App Store. They reported that opinions on product quality formed a large portion of reviews, but opinions on service quality had a bigger effect on sales.

Sentiment analysis has identified frequent causes of user complaints, and has helped to prioritise informative reviews. on the dataset used in their earlier study [88]. Liang et

Malavolta et al. [147], [148] analysed 3 million reviews from 11,917 Google Play apps, and produced a summary of user perceptions about 445 hybrid apps [94] compared with native apps. The authors found that hybrid mobile apps received similar ratings to native apps, but native apps had been reviewed on average 6.5 times more. They planned (at the time of writing) to replicate the work using multiple stores and a small set of cross-platform apps, to compare their perception across different platforms. Vu et al. [233], [234] developed MARK, a system that identifies keywords in sets of reviews in order to assist with summarisation and search. The method is one of several summarisation approaches that are applied to reviews.

$\mathrm{Gu}$ and Kim [83] proposed SUR-Miner, a review summarisation and categorisation tool, that they evaluated on 2,000 sentences mined from reviews of 17 Google Play apps. The tool was intended for use by developers, and produces a visualisation of the reviews. The authors surveyed the developers of the studied apps, of whom 28 out of 32 agreed that the tool is useful.

Review summarisation helps developers to gather information from large numbers of reviews that would be infeasible to read individually. A number of tools have been produced, such as WisCom, AR-Tracker, MARK and SUR-Miner.

\subsection{Surveys and Methodological Aspects of App Store Analysis}

Martin et al. [154] identified the App Sampling Problem, finding that the majority of past work used partial subsets of biased data for app review analysis. The authors assessed the bias and identified techniques that can be used to ameliorate its effects, as well as defining a classification scheme that can be applied to app review analysis studies to describe dataset completeness. Khalid et al. [118] reviewed recent literature in app store review analysis, and made several suggestions that could improve the app reviewing process for both users and developers. They suggested that the process could be improved by assigning categories to reviews, and adding sort and filtering functionality based on the assigned category, helpfulness and star rating. The authors also suggest that adding a user reply feature would assist the developers to get the highest quality reviews. associated 'complaint' type about price, that free apps did not.

In 2015 McIlroy et al. [160] studied reviews in Google Play and Apple App Store, and developed an automated labelling scheme that can identify multiple elements to reviews that could be beneficial to stakeholders. For example, a review might contain a feature request and a bug report, and so a label for each type would be applied to it. Gao et al. [70] proposed AR-Tracker, a similar tool to AR-Miner [41], that automatically collects user reviews of apps and ranks them in order to optimise the representation of the review set, in terms of frequency and importance. Pérez [228] mined and labelled 160 user reviews from 5 Google Play apps in order to train a review categorisation tool, that identifies feature requests and bug reports. The tool was evaluated on 400 labelled reviews and achieved an 0.78 accuracy.
There have been two recent methodological analytical surveys of the review analysis literature, including suggestions for improving the reviewing process, and the highlighting of a prevalent methodological issue called the "App Sampling Problem".

\subsection{Future Work}

Many studies have produced tools which can aid in the summarisation and requirements extraction from reviews, but these tools have not been widely adopted as of yet by developers. Future work may seek to bridge this gap, by making tools available to developers in some form. 
TABLE 7

Chronological summary of security-related App Store Analysis literature showing the authors, publication year, store used: $g$ signifies Google Play or other Android stores, or the Android platform in general, and a signifies Apple App Store; the type of literature, and the number of apps used in the study.

\begin{tabular}{|c|c|c|c|}
\hline Authors [Ref], Year & Store & Venue & No. apps \\
\hline Blasing et al. [27], 2010 & $\mathrm{~g}$ & MALWARE & 150 \\
\hline Batyuk et al. [20], 2011 & $\mathrm{~g}$ & MALWARE & 1,865 \\
\hline Potharaju et al. [183], 2012 & $\mathrm{~g}$ & ESSoS & 158,000 \\
\hline Moller et al. [170], 2012 & $\mathrm{~g}$ & LARGE & 1 \\
\hline Chia et al. [43], 2012 & g & WWW & 19,344 \\
\hline Gibler et al. [72], 2012 & $\mathrm{~g}$ & TRUST & 24,350 \\
\hline Grace et al. [81], 2012 & $\mathrm{~g}$ & WiSec & 100,000 \\
\hline Crussell et al. [50], 2012 & $\mathrm{~g}$ & ESORICS & 75,000 \\
\hline Peng et al. [181], 2012 & $\mathrm{~g}$ & CCS & 500,000 \\
\hline Zhu et al. [262], 2015 & g & ICICS & 5,685 \\
\hline Awang Abu Bakar & $\begin{array}{l}8 \\
g\end{array}$ & ACSAT & 5,000 \\
\hline \multicolumn{4}{|l|}{ Mahmud [14], 2013} \\
\hline Stevens et al. [211], 2013 & $\mathrm{~g}$ & MSR & 10,300 \\
\hline Book et al. [29], 2013 & $\mathrm{~g}$ & CoRR & 114,000 \\
\hline Sanz et al. [198], 2013 & $\mathrm{~g}$ & Cybernet. Syst. & 333 \\
\hline Sanz et al. [200], 2013 & g & SECRYPT & 333 \\
\hline Sanz et al. [199], 2013 & $\mathrm{~g}$ & NSS & 333 \\
\hline Wang et al. [240], 2013 & $\mathrm{~g}$ & DBSec & 272,774 \\
\hline Crussell et al. [51], 2013 & $\mathrm{~g}$ & ESORICS & 265,359 \\
\hline Gibler et al. [73], 2013 & $\mathrm{~g}$ & MobiSys & 265,359 \\
\hline Peiravian & $\mathrm{g}$ & ICTAI & 1,250 \\
\hline \multicolumn{4}{|l|}{ Xingquan [180],'13 } \\
\hline Chakradeo et al. [37], 2013 & $\mathrm{~g}$ & WiSec & 14,888 \\
\hline Pandita et al. [177], 2013 & g & SEC & 581 \\
\hline Zhu et al. [259], 2013 & $\begin{array}{l}\circ \\
\mathrm{a}\end{array}$ & CIKM & 15,045 \\
\hline Liu et al. [143], 2014 & $\mathrm{w}$ & NSDI & 51,150 \\
\hline Crussell et al. [52], 2014 & $\mathrm{~g}$ & MobiSys & 165,426 \\
\hline Gorla et al. [79], 2014 & $\mathrm{~g}$ & ICSE & 32,136 \\
\hline Zhang et al. [250], 2014 & $\mathrm{~g}$ & WiSec & 10,311 \\
\hline Dering and McDaniel [57], 2014 & $\mathrm{~g}$ & MILCOM & 450,000 \\
\hline Ham and Lee [90], 2014 & g & IJCCE & 10 \\
\hline Bhoraskar et al. [26], 2014 & $\mathrm{~g}$ & SEC & 1,010 \\
\hline Qu et al. [186], 2014 & $\begin{array}{l}\circ \\
g\end{array}$ & CCS & 45,811 \\
\hline Zhu et al. [261], 2015 & a & TKDE & 15,045 \\
\hline Kim et al. [121], 2015 & $\mathrm{~g}$ & ASE & 350 \\
\hline Wang et al. [237], 2015 & $\mathrm{~g}$ & ISSTA & 105,299 \\
\hline Schütte et al. [203], 2015 & $\mathrm{~g}$ & ConDroid & 10,000 \\
\hline Mutchler et al. [169], 2015 & $\begin{array}{l}0 \\
g\end{array}$ & MoST & 998,286 \\
\hline Avdiienko et al. [13], 2015 & $\begin{array}{l}\circ \\
g\end{array}$ & ICSE & 2,866 \\
\hline Ma et al. [145], 2015 & $\mathrm{~g}$ & COMPSAC & 22,555 \\
\hline Vigneri et al. [227], 2015 & $\mathrm{~g}$ & CoRR & 5,000 \\
\hline Yang et al. [247], 2015 & $\mathrm{~g}$ & ICSE & 633 \\
\hline Lageman et al. [124], 2015 & $\mathrm{~g}$ & MILCOM & 417 \\
\hline Deng et al. [56], 2015 & a & CCS & 2,019 \\
\hline Zhang et al. [251], 2015 & g & CCS & 100 \\
\hline Huang et al. [102], 2015 & $\mathrm{~g}$ & SEC & 16,000 \\
\hline \multirow[t]{3}{*}{ Chen et al. [39], 2015} & $\mathrm{~g}$ & SEC & $1,165,847$ \\
\hline & & Mea & 106,929 \\
\hline & & Median & 14,888 \\
\hline
\end{tabular}

An avenue of research that has not been attempted is the study of reviews in the Windows Phone Store, which was launched in 2010 but has not achieved the widespread success of Google Play and Apple App Store, in the competitive market. In particular, a comparison of the review taxonomy, system and culture between different platforms including Windows Phone store is a potential future work.

\section{Security}

Studies relating to app security are discussed in this section, and are summarised in Table 7. We can see from Table 7 that the number of studies grew year on year until 2013 and then remained stable. A large proportion of these papers do not combine technical with non-technical attributes. Instead, they use properties such as the validation that highly rated apps have received, through being downloaded, used, and highly rated by many users. Much of the security-related literature uses the property that popular apps can generally be assumed to be non-malware, since they are scanned prior to being hosted in the store, and have large user bases.

There are many studies on mobile app security that use app stores in a less direct way than those discussed in this section, some of which are mentioned in Section 13.2. Additionally, literature in Section 6.4 has identified potential risks associated with permissions, beyond the more direct security threats discussed in this section.

Many studies in this section use large collections ( $>10,000$ apps) of benign apps to help distinguish between benign and malicious behaviour. The number of apps used ranges from 1 to 1,165,847 (which also happens to be the largest study in this survey).

Security literature is broken down into "Faults", "Malware", "Permissions", "Plagiarism", "Privacy" and "Vulnerability" subsections.

\subsection{Faults}

Ravindranath et al. [188] used a sample of apps mined from Windows Phone Store to run their greybox fault detection tool. They found that 1,138 of the sample of 3,000 apps had failures. Liu et al. [143] presented their DECAF system for detecting advertisement placement and layout violations, that can be used to indicate advertisement fraud. They tested the system on 51,150 Windows apps for tablet or phone, and plan to extend it to detect more types of rule violation. The DECAF system was used by Microsoft Advertising in 2013 to prompt developers to comply with layout rules.

Crussell et al. [52] presented MAdFraud, a system that detects advertisement fraud in the form of requesting ads while the application is in the background, and in the form of simulating user clicks on advertisements. They tested the system on 165,426 apps gathered from Google Play and a separate security company, and found that $30 \%$ of apps made advertisement requests while running in the background, and 27 apps simulated user clicks on advertisements. Deng et al. [56] introduced their iRis system, that performs static analysis on iOS apps in order to detect suspicious apps that may violate Apple's terms of service. The authors detected 146 apps from a sample of 2019, that accessed sensitive user information through use of private APIs.

Faults in mobile applications can point to potential security risks. Work in fault analysis has detected layout rule violations, terms of service violations and advertisement library issues.

\subsection{Malware}

In 2010 Bläsing et al. [27] used the top 150 free Google Play apps to test their static and dynamic APK analyser. 
They tested these apps against 1 known malware app, which was shown to be an outlier, establishing that their approach has the potential for malware detection. Peng et al. [181] proposed an app risk rating system trained on metadata from name, category and set of permissions. The system was trained on a set of 378 malware apps, and evaluated on almost 500,000 apps mined from Google Play. Zhu et al. [262] proposed an approach to malware detection that uses permission and description information to detect abnormal permission sets. They evaluated the system on 5,685 apps mined from Google Play and found some words that had a large effect on permission validity; they also tested the system on known malware and found that it was able to successfully detect it as such.

Chakradeo et al. [37] created an app malware triaging tool call MAST, that they trained on known malware and a set of 14,888 apps mined from Google Play (that were assumed to be benign). Peiravian and Xingquan [180] trained a malware classifier using 1,250 samples of known malware, and 1,250 samples of (assumed benign) apps mined from Google Play. They trained the classifier using information on the permissions requested and the API calls made by the apps.

Sanz et al. [200] used cosine similarity between the sets of features declared in Android manifest files, in order to detect anomalies that might suggest the presence of malware when compared with a benign set. Sanz et al. later trained machine learning classifiers to distinguish between sets of known malware and 333 benign apps mined from Google Play [198], [199]. Similarly, Wang et al. [240] proposed DroidRisk, an app trained on sets of known malware and assumed benign software mined from Google Play. DroidRisk rates the security risk of other apps in order to help prevent users from installing malware unknowingly. Apps mined from Google Play are assumed benign as Google's tool Google Bouncer [5] is run to detect malware and remove it from the store.

As a means of detecting potentially malicious apps, Gorla et al. [79] performed topic modelling on app descriptions, and then applied K-means clustering to the results to form distinct clusters. Utilising API information from the app manifest, the authors trained a one-class support vector machine (SVM) [149] on each cluster to detect outliers in terms of API usage. This method indicated apps that exhibit sufficiently different behaviour from the norm to suggest the presence of malware. This approach was later extended by Ma et al. [145] who used known malware and benignware to train their model, and reported improvements on the resultant precision, recall and F-measure.

Avdiienko et al. [13] extracted information flow data from apps in order to train a benign-trained malware classifier. The classifier was trained on 2,950 of the most popular Google Play apps, which were to be assumed benign as their download ranks were in the top 100 in each of 30 categories. In this way, the authors combined non-technical information (download rank) with extracted technical information (information flow) to detect malware. The system reported high precision on sets of known malware from the Genome project [253] and VirusShare database [229]. In a similar way, the 2013 study by Sanz et al. [198] trained machine learning classifiers to separate known malware and benign apps mined from Google Play. The 2014 study on identifying malicious apps using system call events, by Ham and Lee [90], also used apps from the Google Play Games category as a benign set, against which to compare.

Lageman et al. [124] generated feature sets to be used for classification of malware and benignware, from runtime log datasets of 419 malware apps and 417 mined benign apps. They tested the feature set and achieved a true positive rate of $90 \%$ with a Random Forest classifier [76]. In the largest app study to date, Chen et al. [39] ran their DiffCom system on 1,165,847 apps mined from Google Play and third party Android stores. DiffCom detects malware, including zeroday malware, without prior knowledge of malware, using a simple comparison with known apps in the corpus. The system was tested on a sample of 50,000 apps and achieved a false positive rate of 0.04 and false negative rate of 0.06 . When run on the entire dataset, DiffCom detected 127,429 instances of malware and 20 likely instances of zero-day malware.

There has been much work on malware detection through the use of static and dynamic analysis, and also alternative sources of information such as descriptions, API usage and data flow.

\subsection{Permissions}

In 2013 Awang Abu Bakar and Mahmud [14] mined 5,000 apps from the Google Play store and analysed their permissions. They found significant correlations of rho $0.13,0.24$ and -0.13 between (technical) the number of permissions asked for and (non-technical) the price, download rank and rating, respectively. They highlighted the top permissions requested by apps, and found that $40 \%$ of the apps requested the phone's status and identity, a source of sensitive information. Stevens et al. [211] mined 10,300 apps from several Android stores including Google Play, and applied the permissions analysis tool Stowaway [12], that can detect declared and used permissions. The authors found that 44\% of apps in their sample contained at least one unnecessary permission, and computed a Spearman's correlation coefficient of 0.72 between the 'popularity' of permissions on Stackoverflow and their misuse. Book et al. [29] studied library permissions on 114,000 apps mined from the Google Play store, and showed that libraries bundled with apps lead to old versions being included. Increasingly, advertisement libraries were taking advantage of app permissions, presenting a potential security risk that the authors argue should be solved by the app store or privacy legislation.

Pandita et al. [177] presented the WHYPER system for automatically extracting the reason a permission is used from the description. They evaluated the system using 581 apps mined from Google Play, that were manually labelled by the authors. The authors tested the system on the permissions for address book, calendar and audio recording, and achieved an average precision of $82.2 \%$ and recall of $81.5 \%$. In a related study, Qu et al. [186] introduced AutoCog, a tool for checking the fidelity between app descriptions and requested permissions. The authors tested the system on 45,811 Google Play apps, and achieved a precision of $92.6 \%$ and a recall of 92.0\% when detecting 11 permissions. 
The findings by Dering and McDaniel [57] suggest that library usage presents a security risk due to permissions usage. This is discussed in more detail in Section 6.

Library usage can present a security risk by taking advantage of requested app permissions. Tools such as Stowaway, WHYPER and AutoCog check permission usage, comparing it against permissions requested.

\subsection{Plagiarism}

In 2012 Potharaju et al. [183] conducted a study on 158,000 free Android apps, identifying apps that are likely to be plagiarised in order to spread malware. The authors found that the $29.4 \%$ of apps with the most permissive rights were the most likely to spread malware, and that non-technical information such as category, number of downloads and publishing day could increase the initial spread of the malware. Crussell et al. [50] introduced the tool DNADroid, which they used to identify 141 cloned apps in the Google Play store, from a mined set of 75,000 apps. The authors then introduced the tool AnDarwin, that decompiles apps and compares them to detect clones [51]. They detected 4,295 cloned apps using this approach from a mined set of 265,239 apps. This dataset was used in the study by Gibler et al. [73], who investigated the effects of application plagiarism on developers.

Zhu et al. [259], [261] mined ranking, rating and review data from 15,045 apps from the Apple App Store. They detected outliers using hypothesis tests in order to find potentially fraudulent apps. They took a unique approach to the issue with app ranks (only the top apps in Google Play, Windows Phone Store and Apple App Store have download ranks), in that they termed the period in which an app has a rank as a 'leading event' and consecutive events as a 'leading session'. Several authors used API information to detect plagiarised apps [121], [237], [250], whose studies are discussed in more detail in Section 6.

App plagiarism is a common approach to spread malware; a number of authors have detected cloned apps in the Google Play store. Potentially fraudulent apps have also been detected in the Apple App Store.

\subsection{Privacy}

In 2011 Batyuk et al. [20] used the top 1,865 free Google Play apps to test their static APK analyser, which detected that 167 apps accessed private identifiers, thereby presenting a security risk. Of these apps, 114 wrote the information after reading it, which might indicate that the apps contain spyware. The work has since been extended into a static analysis tool called Androlyzer [53]. Chia et al. [43] evaluated the ratings of apps from Facebook, Chrome and Google Play, as a means of warning against privacy risks. They found a strong correlation between popularity and the number of ratings apps had received, but no correlations between permissions sought and privacy risk, nor rating. This result shows that ratings were not an effective indicator of the privacy of apps, and new suspicious apps were not likely to receive many ratings which could have served as a warning for future users.

Gibler et al. [72] mapped Android API calls to privacy information, and performed static analysis to identify apps where private data is leaked. Using their tool, AndroidLeaks, they analysed 24,350 apps from Google Play and third party stores, and found 2,342 apps with privacy leaks. Grace et al. [81] introduced AdRisk, a static analysis tool for identifying potential privacy risks associated with advertisement libraries. From their study on 100,000 apps mined from Google Play, the authors found that 52,067 apps used advertisement libraries, of which $31 \%$ used more than one. The authors remarked that the majority of the 100 studied advertisement libraries were found to collect personal information.

Vigneri et al. [227] used a set of 5,000 apps mined from Google Play, on which they performed dynamic execution to determine network usage. They focused, in particular, on network activity to URLs that they claimed could present privacy or security risks, such as those associated with tracking, spyware or malware. Network activity was compared both within category and overall, in order to determine apps with suspiciously high activity. The authors noted that a large proportion of apps, even those with high ratings and download ranks, downloaded a large number of advertisements. Huang et al. [102] presented their SUPOR system, which detects privacy information entry fields as potential privacy or security risks, using static analysis. They evaluated the system on 16,000 apps mined from Google Play, and obtained a precision of 0.973 and a recall of 0.973 , with a false positive rate of 0.087 . The cases found included fields for national ID, username, password, credit card and health data.

Ratings were not found to correlate with permissions or privacy risk, but suspicious apps did not receive many ratings. Many advertisement libraries have been found to collect personal information, presenting a potent privacy risk.

\subsection{Vulnerability}

Moller et al. [170] studied the update behaviour of users following recent updates, finding from a case study that approximately half of users did not update their app for at least a week after the update. The authors argued that this could have lead to users continuing to run vulnerable software even after a fix was available.

In 2015, Zhang et al. [251] argued that the descriptions given to apps contain insufficient security information. The authors presented the DescribeMe system, which generates security-centric descriptions using static analysis. They performed a user study using Amazon's Mechanical Turk [7], on a set of 100 apps, and asked whether the generated descriptions were readable and whether they could reduce the rate at which users download malware. The generated descriptions achieved a $4 \%$ lower readability rating than the original human-written descriptions, but decreased the malware download rate by 39\%. Yang et al. [247] used 633 apps mined from Google Play as the benign set to test their tool for distinguishing between malicious and benign apps. 
They found that the intent of security accesses was more related to whether an app was malicious than the type of security-sensitive resources that it accessed.

Schütte et al. [203] tested their dynamic analysis tool ConDroid on the top 10,000 free Google Play apps and found 172 apps suffered from a logic bomb vulnerability, by selectively executing code sections that use vulnerable APIs. Mutchler et al. [169] took a snapshot of 1,172,610 Google Play apps. They found that 998,286 of these apps used the WebView API, indicating that the apps used an embedded WebView in some way. The authors searched for several known vulnerabilities and found that $28 \%$ of the studied apps had at least one of these vulnerabilities. As a result, the authors proposed a set of API changes to mitigate such threats. In a related study, Bhoraskar et al. [26] mined 1,010 apps from Google Play and used static analysis and partial app rewriting to check for known security issues in third party components. They found that 13 of 200 apps using the Facebook SDK were vulnerable to known attacks, and that 175 of 220 children's apps potentially collected information in violation of the US Children's Online Privacy Protection Act [46].

Reading app descriptions before downloading them can help to protect against vulnerability, since machinegenerated descriptions are less readable, thereby service as potential warnings. Several authors have identified prevalent vulnerabilities, such as logic bombs and embedded WebViews.

\subsection{Future Work}

There is potential future work in App Store Security Analysis in augmenting approaches with the non-technical information made available by app stores. Additionally, there is a great deal of literature on app security, perhaps warranting a standalone survey that can bring together elements that do not meet the scope for app store analysis.

The majority of studies in the section use datasets from Google Play, three study Apple datasets and just one studies Windows. Potential future work may therefore seek to study security on collections mined from Apple, Windows and Blackberry, and may extend to cross-store security analysis or comparison.

\section{Store Ecosystem}

In this section we discuss literature that focuses on a store's ecosystem, or the differences between stores. This literature is summarised in Table 8.

The scale of store ecosystem studies ranges from 1 to $1,164,489$ apps, and simulations have included up to 1,250,000 apps.

Store Ecosystem literature is broken down into "Interstore", "Intra-store", "Recommendation" and "Simulation" subsection.

\section{TABLE 8}

Chronological summary of store ecosystem-related App Store Analysis literature showing the authors, publication year, store used: g signifies Google Play or other Android stores, a signifies Apple App Store, $b$ signifies the Blackberry store and $w$ signifies Windows Phone; publication venue, and the number of apps used in the study. Numbers in the table indicate empirical app data mined from stores, $\left(^{*}\right)$ signifies 500,000 simulated apps, $\left(^{* *}\right)$ signifies $1,250,000$ simulated apps, and $\left({ }^{* *}\right)$ signifies over 500 simulated apps (final values were not specified by the paper's authors); only empirical data is considered for mean and median.

\begin{tabular}{lllr}
\hline Authors [Ref], Year & Store & Venue & No. apps \\
\hline Syer et al. [213], 2011 & b,g & SCAM & 3 \\
d'Heureuse et al. [58], 2012 & a,b,g,w & MCCR & $1,164,489$ \\
Jung et al. [113], 2012 & a & Market Lett & 1,189 \\
Lim and Bentley [131], 2012 & a & GECCO & $*$ \\
Lim and Bentley [130], 2012 & a & ALIFE & $*$ \\
Lim and Bentley [132], 2013 & a & CEC & $*$ \\
Garg and Telang [71], 2013 & a & MIS & 1,223 \\
Ihm et al. [107], 2013 & $\mathrm{g}$ & CGC & 10 \\
Zhong \& Michahelles [252],'13 & $\mathrm{g}$ & SAC & 191,301 \\
Petsas et al. [182], 2013 & $\mathrm{g}$ & IMC & 316,143 \\
Syer et al. [215], 2013 & $\mathrm{g}$ & CASCON & 15 \\
McDonnell et al. [158], 2013 & $\mathrm{g}$ & ICSM & 10 \\
Cocco et al. [44], 2014 & $\mathrm{a}$ & MWIS & $* * *$ \\
Wenxuan and Airu [243],'14 & $\mathrm{a}, \mathrm{g}, \mathrm{w}$ & ICDMW & 736,377 \\
Ng et al. [173], 2014 & $\mathrm{g}$ & COMPSAC & 506 \\
Liu et al. [141], 2015 & $\mathrm{g}$ & WSDM & 6,157 \\
Ruiz et al. [165], 2015 & $\mathrm{g}$ & IEE Soft. & 10,150 \\
Syer et al. [214], 2015 & $\mathrm{g}$ & Soft. Qual. & 5 \\
Joorabchi et al. [112], 2015 & $\mathrm{a}, \mathrm{g}$ & ISSRE & 14 \\
Gómez et al. [77], 2015 & $\mathrm{g}$ & ICSE NIER & 1 \\
Askalidis [11], 2015 & $\mathrm{a}$ & CoRR & 162 \\
Xie and Zhu [245], 2015 & $\mathrm{a}$ & WiSec & 179,353 \\
Corral and Fronza [47], 2015 & $\mathrm{g}$ & MOBILESoft & 100 \\
Lim et al. [133], 2015 & $\mathrm{a}$ & TEVC & $* * *$ \\
\hline & & Mean & 144,845 \\
& & Median & 848 \\
\hline
\end{tabular}

\subsection{Inter-store}

In 2011, Syer et al. [213] studied the different code practices between app stores, by selecting 3 pairs of feature-equivalent apps from Android and Blackberry. The authors analysed the source code, code dependencies and code churn of these apps, and found that the Android apps were generally smaller but rely heavily on the platform. Conversely, Blackberry apps were larger and relied heavily on 3rd-party APIs. In order to reach the largest customer base developers need to cater for each platform, and so the authors remarked that it is therefore easier to develop for Blackberry and port to Android than vice versa. Syer et al. [215] later compared development practices between 15 Android apps and 5 traditional desktop and server applications. They found that mobile apps were most similar to Unix utilities, in terms of smaller code bases and small development teams. However, they also reported that mobile apps suffered from greater numbers of defects and slower fix times than the studied traditional applications.

In 2012 d'Heureuse et al. [58] mined 1,164,489 apps in total from Apple, Blackberry, Google and Windows app stores. The apps were mined at regular intervals over a period of 3 months, in order to perform cross store comparison and also to study growth over time. The authors found that the smaller stores (Blackberry and Windows) had similar rates of growth to the larger stores (Apple and Google), at 2\%. The smaller stores (Blackberry and Windows) were found to be the most expensive, and all stores displayed a similar power-law curve 
in price, with many cheap and free apps. Apps that appeared in multiple markets were on average 7.15 MB larger in the Apple store, and were a similar size in the 3 other stores.

Petsas et al. [182] analysed the downloads of 316,143 apps from 4 third-party Android app stores. They found that $10 \%$ of the apps accounted for at least $70 \%$ of the total downloads in the stores, and that user downloads followed a clustering type behaviour, where their subsequent app downloads were usually in the same category. The authors also found that popularity followed a power-law distribution against app price, for paid apps. $\mathrm{Ng}$ et al. [173] looked into the safety of third-party Android stores by downloading the top apps from Google Play and 20 other third-party Chinese Android app stores. They compared the APKs to check whether they were the same as the official releases, and ranked the severity level of differences. The authors concluded that the third party app stores studied could not be trusted, as the proportion of apps which did not match their official releases was high, as were the corresponding difference severity levels.

In 2015 Ruiz et al. [165] conducted a longitudinal rating study on 10,150 apps over the period of 12 months. They argued that the Amazon style rating system, in which ratings are accumulated over the lifespan of an app, is too slow to adapt to changes in apps, whose performance is determined by the current release. The current Google Play rating system makes it more difficult for an app to increase its rating with a strong release than, for example, the Apple App Store rating system. Joorabchi et al. [112] introduced CheckCAMP, a tool that checks for inconsistencies between Android and iOS versions of the same app. The authors tested the tool on 7 open source apps and 7 industry apps, and validated their results with a user study, finding an F-measure of 1.0 on the open source apps and an F-measure of 0.92 on the industry apps.

Inter-store analysis literature has compared code practices, growth, user download behaviour and consistency between app stores such as Google, Blackberry, Apple and Windows. Third party Android app stores have been found to host apps that appear identical yet contain significant differences to the official versions, a sign that they may contain malware.

There are sources of non-technical information that replicate information found on app stores, but provide a more accessible means to gather the data. For example, the study by Syer et al. [214] uses information on the number of downloads from AppBrain, a replication of the number of installs bracket on Google Play (eg. 1,000,000 - 5,000,000 installs appears on AppBrain as $1,000,000+)$. Ihm et al. [107] combined download information on 10 social networking apps from Google Play with the number of registered users on their respective websites. They found a strong correlation between the two metrics.

\subsection{Intra-store}

Jung et al. [113] assessed the differences between free and paid apps on Apple's Korean App Store. They found that customer ratings were more critical to the survival of free apps, and there is also benefit to getting an early entrant in markets. In 2013 McDonnell et al. [158] studied 10 apps using source code from github [75]. The Android platform was shown to be evolving, with an average of 115 API updates per month, due to which $28 \%$ of Android references were out of date, and the median lag time to update to support a new API was found to be 16 months. The APIs used most were the ones updated most frequently, yet interestingly API updates were more defect prone than other changes in client code.

Apps in Google Play do not (at the time of writing) have accessible information on their number of downloads, other than 'ranges', such as the range 50-100. Zhong and Michahelles [252] analysed the distributions of download buckets and ratings of 191,301 apps from Google Play. They found that a small number of popular 'blockbuster' apps accounted for the majority of app downloads, and also had high ratings indicating customer satisfaction. Paid apps achieved more success when they were cheaper, but expensive professional apps had disproportionally high numbers of downloads. The authors concluded that developers could break into the higher download ranking positions by fulfilling a niche market. Garg and Telang [71] compared paid app demand in the Apple App Store, using download ranks. They found that the top ranked paid app is downloaded 120-150 times more than the 200th ranked app.

Askalidis [11] studied the effects of sales promotions in the Apple App Store on 162 apps. They found that rival apps were able to benefit from a promotion, so long as their promoted price was cheaper than their competition. They authors also found that sales where apps became free, or had easily redeemable digital discounts, were the most successful. Sales were shown to have mixed effects on the ratings of apps. Gómez et al. [77] proposed an app store feature of automatically patching defective apps, which they demonstrated by automatically fixing a defective app mined from Google Play. Xie and Zhu [245] investigated the practice of promoting apps through buying positive reviews, via illicit "underground" services. The authors registered on 8 such app promotion sites and exposed approximately 30,000 promoted apps. Their tool, AppWatcher, was used to collect information from 179,353 randomly selected iOS apps, from which they mined 9,399,014 reviews. The authors reported on differences between datasets of promoted and random apps.

Corral and Fronza [47] studied 100 open source apps that are available on the Google Play store. They performed correlation and regression analyses between source code quality metrics and the store performance metrics number of downloads, number of reviewers and average rating. The authors found no strong correlation and no strong regression coefficients, rejecting their initial hypotheses that source code quality plays a role in app success.

Source code quality has been shown to have no strong correlation to app success for open source apps. However, factors such as price, sales promotions and catering to a niche market may all play a factor in app success. 


\subsection{Recommendation}

In 2014 Wenxuan and Airu [243] used information on the number of downloads and numbers of reviews, as well as the numbers of apps downloaded by and reviewed by participating users. This data was used as part of a recommendation system called Interoperability-Enriched Recommendation (IER), which enables them to recommend similar apps to a user in the Windows Phone Store using data mined from 736,377 Google Play, Apple App Store, and Amazon App Store apps. Liu et al. [141] also studied app recommendation systems, by incorporating the level of privacy that the app needs as well as user interests. They evaluated their approach using 6,157 apps mined from Google Play, and found that their recommender performed better when treating each app function with different privacy allowances. They used the rating distribution over their dataset as the motivation for modelling user preference with a Poisson distribution.

The Interoperability-Enriched Recommendation (IER) system uses cross-store data to make app recommendations. Privacy allowances have also been used to enhance app recommendation for Google Play.

\subsection{Simulations}

Lim and Bentley simulated the app store ecosystem using an agent-based evolutionary model, in order to experiment with different publicity strategies [130], [131], modelling apps with infectious properties, so that they can spread after being downloaded by a user. They found that an 'app epidemic' is most likely to occur when the app appears on the 'new apps' chart. The authors then used the model to explore different ranking algorithms [132]. The study simulated users, and experimented with alternating time periods for updating the "new apps" chart, and the degree to which historical performance factors into the "top apps" chart. The study found that the top apps chart performed best in terms of overall downloads by incorporating fresh apps, and for this to work it needed to incorporate less historical performance data (also found later by Ruiz et al. [165]).

Lim et al. later simulated the ecosystem from a user's perspective [133], using collected usage information from over 10,000 participants [129]. They modelled developer strategies such as 'innovator' (who produces apps with random features) and 'copycat' (who copies the app) [133]. They found that 'optimiser' (who improves on the original 'innovator' apps) and 'copycat' working together led to the best overall fitness, provided that they represented a low proportion of the overall modelled developer population.

An agent-based evolutionary model has been used by multiple authors to simulate an app store. Authors have studied such factors as ranking algorithms and the optimal interaction between simulated developers.

Cocco et al. [44] extended the model used by Lim and Bentley, and investigated additional ranking algorithms and user behaviour. They explored store ranking algorithms, and
TABLE 9

Chronological summary of App Store Analysis literature related to size and effort prediction showing the authors, publication year, publication venue, and the number of apps used in the study.

\begin{tabular}{llr}
\hline Authors [Ref], Year & Venue & No. apps \\
\hline Sethumadhavan [206],'11 & ISMA & 6 \\
Preuss [185], 2012 & The IFPUG Guide to IT \& & 1 \\
& Software Measurement & \\
Preuss [184], 2013 & ICEAA & 1 \\
van Heeringen and van & IWSM-MENSURA & 0 \\
Gorp [223], 2014 & & \\
Abdullah et al. [1],'14 & ICOS & 0 \\
D'Avanzo et al. [55],'15 & SAC & 8 \\
Francese et al. [66],'15 & SEAA & 23 \\
Ferrucci et al. [63],'15 & SEAA & 13 \\
Ferrucci et al. [64], 2015 & PROFES & 13 \\
\hline & Mean & 7 \\
& Median & 6 \\
\hline
\end{tabular}

found that a $1 \%$ chance of a new app appearing in the top charts leads to the highest downloads-to-browse ratio.

\subsection{Future Work}

There are potential research opportunities to be found comparing stores, especially comparing the Windows Phone Store against more well-studied stores such as Apple, Blackberry and Google. Future studies may continue to build on the store simulation work by Lim et al., and may extend analysis to the success or evolution of less widely used stores such as Windows or Blackberry.

\section{Size and Effort Prediction}

Papers that predict size or effort based on the functionalities offered by an app are discussed in this section, and are summarised in Table 9. Many of the papers mine apps from Google Play, and compare the resultant predicted size with the actual size reported in the store and/or LOC (number of Lines Of Code) of the apps.

The scale of size and effort prediction studies is relatively small but, since the field has witnessed strong growth in 2015, it seems likely that the scale of studies will grow in the future.

In 2011 Sethumadhavan [206] discussed the application of Function Point Analysis (FPA) to Android applications, pointing out that compared with traditional desktop applications, mobile apps contain limited functionality, and often functionality is merely a wrapper to system functionality. Preuss [184], [185] then showed how FPA can be used for the estimation of the cost of a mobile app, using the approach on a case study Android application. In 2014 van Heeringen and van Gorp [223] discussed how to use COSMIC [48], a second generation functional size measurement method, in order to measure the functional size of mobile apps. Abdullah et al. [1] discussed using the COSMIC method to estimate game apps, using an intermediate representation of required assets and functionality in the Unity3D game engine.

In 2015 D'Avanzo et al. [55] applied the COSMIC approach to 8 Google Play apps, and applied linear regression 
to the functional point size in order to estimate the code size. By applying leave-one-out cross validation, the authors showed that the approach could accurately estimate code size based on functionality alone, once trained. Francese et al. [66] used linear regression to estimate the development effort needed, and the number of GUI components, based on requirements alone. The authors found, from a study on 23 Android applications, that the estimates were accurate when trained on source code metrics such as classes, files and LOC. Ferrucci et al. [63] applied the COSMIC approach to 13 Android applications, and showed that functional size was strongly correlated with app size, and that it could be used to accurately estimate the bytecode size of an app. Ferrucci et al. [64] later compared the related approaches by D'Avanzo et al. [55] and van Heeringen and van Gorp [223] on their dataset of 13 Android apps. They found that functional size results were correlated with multiple app size measures, but that the approach presented by D'Avanzo et al. [55] was more accurate.

Function Point Analysis (FPA) and the COSMIC method of measuring the functional size of apps, have been used to predict the size and effort of apps.

\subsection{Future Work}

Size and effort prediction is a relatively small section of app store analysis, that we expect to continue to grow. Future studies may extend to Apple, Windows and Blackberry stores, and may seek to incorporate mined feature or API information to increase their prediction accuracy. We also expect predictive modelling to be used for estimating other properties of mobile apps, such as faults and crashes.

\section{Closely Related Work}

The following literature is important to the field of App Store Analysis, yet does not quite fully meet our exact definition of App Store Analysis. Nevertheless, we recognise that it would be restrictive to apply our definition too rigorously at this early stage in App Store Analysis. Furthermore, since this work meets aspects of our definition we regard it as closely related. We do not claim to comprehensively survey this literature, but provide it to add context to the App Store Analysis literature discussed thus far in the survey.

\subsection{User Surveys and Studies}

There is a cross section of App Analysis studies which survey or study user behaviour and feedback, but the information is not specific to observed apps, and is therefore not combined with technical information.

In 2011 Böhmer et al. [28] studied 4,100 Android users for app usage statistics. This was done using AppSensor, an application that monitors the usage of other apps on an Android device. They found that the average application usage session was less than 72 seconds long, and that smartphones were used for almost 60 minutes every day. The type of application was found to differ between times of day, such as news applications in the morning and games at night.
The exceptions to this rule were communication apps, which were used throughout the day. In 2012, Ferreira et al. [62] surveyed 4,035 Android user charging habits, using an app to record their behaviour. Lin et al. [134] conducted a survey on 179 Android users, that asked about their expectations of app purpose and sensitive data handling. They found that the problem of apps not meeting expectations or utilising sensitive data unexpectedly was prevalent, and outlined potential store interface changes to rectify the issue.

Shi et al. [209] developed a mobile app recommendation system that works by learning user preferences. Similarly, Zhu et al. [258] mined context-aware user preferences using log information. Rein and Münch [189] carried out a user study involving mock purchasing for planned app features, in order to determine both the priority and ideal pricing for the features. In 2013, Oh et al. [174] surveyed 100 app users and found that users were more likely to take a passive approach and delete apps rather than reviewing or contacting developers, but when they took an active approach, reviewing was the most popular approach. In 2014 Tan et al. [216] surveyed users and developers of the Apple App Store, regarding the iOS permission request explanation feature. The feature was infrequently used, but the survey found that users would be significantly more likely to accept a permissions request if an explanation was given.

In 2015, Lim et al. [129] surveyed app users from 15 countries to understand how usage of apps and app stores differed by region. They found that behaviour did differ significantly by region in many regards. In Eastern regions, such as China and India, a greater proportion of users participated in recommendation and rating of apps, almost 4 times the proportion of Western users. Additionally, the survey found that app abandonment was higher than average in Brazil and the UK, and lower than average in Japan and France, indicating that differences were influenced by more than global region.

\subsection{Related Security}

We present some of the key app security studies that do not perform App Store Analysis, but that influenced some of the papers described in Section 10.

Enck et al. [60] introduced Kirin, an Android app certification tool for flagging potential malware using a set of rules. In 2010 Enck et al. introduced TaintDroid [59], a tool for tracking the flow of sensitive information within an Android app. TaintDroid was one of the first static analysis tools for Android and was built on extensively in subsequent work. Another information flow extraction tool was created by Arzt et al. [10] in 2014, called FlowDroid. This tool statically analyses information flow to find all possible flows. Mao et al. [151] introduced Sapienz, an Android testing tool that the authors applied to the top 1,000 Google Play apps, revealing 558 previously undetected crashing test causes.

Some authors have used sets mined from Google Play as benign app sets to test against known malware: Xu et al. [246], Rastogi et al. [187], Jing et al. [110], Arp et al. [9], Wang et al. [239], Liu and Liu [144], Roy et al. [191] and Khanmohammadi et al. [120]. Ho et al. [97] used the top 10 most popular apps in each category as a benign set, upon which to test their framework for root kit exploit containment. 
Other authors have used sets mined from app stores to test their tools on large real-world datasets: Barrera et al. [17], Jeon et al. [108], Grace et al. [82], Crussell et al. [50], [51], Ravindranath et al. [188], von Rhein et al. [232], Li et al. [127], Huang et al. [103], Cen et al. [34], Liu et al. [142] and Bastani et al. [19].

\subsection{Reports}

Initial studies, such as the 2010 work by Sharma et al. [208], evaluated the size and growth of the apps market up to the time. In 2011 Butler [31] conducted a study on the Android system, highlighting how it was changing mobile development by enabling people with no prior development experience to release an app. In 2012 Shuler [210] published a report on the Apple App Store Education category, comparing it with their previous study in 2009. They found that over $72 \%$ of the top-selling apps in this category targeted children under 11 years of age, a number that had significantly increased from $47 \%$ in 2009 . Additionally, the average price of an app had risen by 1 USD since 2009, and the majority of top Education developers in 2012 had not been present in 2009.

The 2013 report by Vision Mobile [230] on app industry monetary value and growth found that $72 \%$ of developers are dedicated to Android. iOS and Android developers earned on average double that of developers of other platforms, and iOS was considered the highest priority platform. As of 2013, iOS, Android and Blackberry were the leading platforms, despite Blackberry's decline, and the launch of the prospect Windows Phone Store in late 2010. Vision Mobile have released yearly reports since 2012 on aspects such as developer share, industry revenue and growth. The organisation gathers information by surveying developers worldwide.

\subsection{Mining Tools}

Due to the plethora of analysis and research opportunities presented by app store data, and indeed also due to the difficulties involved with mining app stores, several mining tools have been published.

In 2013, Awang Abu Bakar and Mahmud [16] published OSSGrab, which mines HTML pages from Google Play. The tool was built in order to facilitate their app permissions study [14]. In 2014, Viennot et al. introduced the PlayDrone Google Play crawler [225], to facilitate their large scale API study [226].

The Android Malware Genome Project [253] is a popular source of malware applications for testing security tools. In 2015 Krutz et al. [123] made available a dataset containing 1,179 open source applications. The AndroZoo project provides a large collection of Android APKs [221].

\section{Guidelines for Future App Store AnAL- YSIS AUTHORS}

In this survey we have reported on the general content of studies, as well as the scale of apps used, and the store used. Our previous overview of review analysis [154] synthesised the number of reviews used, and the type of reviews dataset. In future surveys it may be possible to synthesise more information from future literature. Such richer analysis, facilitated by richer data reporting, could lead to new insights and directions in the field of App Store Analysis. We therefore present the following guidelines for data to include, as suggestions for future App Store Analysis work, to help facilitate future studies such as SLRs:

App Stores used to gather collections of apps.

Total number of apps used in the study.

Breakdown of free / paid apps used in the study, including information regarding in-app-purchases where possible.

Categories used, with breakdown of app counts in each category.

Indication of whether API usage was extracted from the studied apps to facilitate the study.

Indication of whether code was needed from apps to facilitate the study.

Indication of whether open source apps were used exclusively for all of part of the study.

Total number of reviews used, if any.

Breakdown of sampling dataset used [154] where applicable, particularly when reviews are used.

Description of ratings and user feedback categories, including trends and response ranges.

Details of statical analysis techniques that were used in the study.

\section{FUTURE WORK}

Here we discuss potential future avenues of research for app store analysis. Other such discussions can be found in the works by Al-Subaihin et al. [3], and Nagappan and Shihab [171].

Expectations: We expect to see the scale of app samples used increase in the years to come, as app stores increase in scale. Google Play and Apple App Store have both exceeded 1.5 million apps, and already there are studies featuring over 1 million apps. We also expect to see more longitudinal studies: the sub-fields for prediction and release engineering studies lend themselves particularly well to longitudinal data, and both of these fields grew in 2015.

As many studies in Section 10 have shown, app cloning and replication is a common problem in app stores. It may be the case that app stores will not grow indefinitely, and may even shrink in size following a consolidation of unique apps present, possibly using the some of the techniques discussed in this survey. In the meantime, as app stores continue to grow, app discovery presents a crucial problem to newer apps or developers, and so we may expect to see an effort to improve discovery, such as a greater-tiered category system. Opportunities: An avenue for future research concerns the extraction of non-technical information from app stores, and extracting samples of apps (cognisant of the App Sampling Problem). Cross-store studies are also an avenue for future research. Few studies have compared multiple app stores, yet there is potential to learn the differences between dominant stores, and lesser known or fledgling stores.

App stores provide us with with the unique opportunity to leverage customer, business and technical aspects of applications in the same place. Future app store analysis studies may seek to further combine all of these aspects to provide greater insights into the socio-technical business of developing for app stores. 
Problems: Restricted data availability in app stores presents issues for researchers. We encourage app store owners not to impose such restrictions as: limiting the ranked list of apps to the top few hundred; limiting reviews to the most recent only. Such restrictions could reduce the scope and accuracy of future App Store Analysis studies. In addition, more detailed breakdowns of the prices attached to free apps that utilise in-app-purchases could lead to valuable research findings for app developers.

A concept that could be extremely valuable to researchers is that of a centralised repository of app store data that can be freely accessed, consisting of apps that are not just "free and open source". However, legal and copyright issues present potent barriers to the construction of such a repository from being created at present, and so this remains an open problem.

\section{CONCLUSIONS}

We have surveyed the published literature in App Store Analysis for software engineering, and identified the key subfields of App Store Analysis to date: "API analysis", "feature analysis", "release engineering", "review analysis", "security analysis", "store ecosystem comparison", and "size and effort prediction". Newer sub-fields such as "release engineering" and "size and effort prediction" have shown strong growth in 2015, suggesting that they might eventually overtake other smaller sub-fields such as "store ecosystem".

The scale of app samples used in studies has increased: in 2015 the number of studies using between 10,000 and 100,000 apps was approximately three times that of 2014. We have observed the emergence of new areas of App Store Analysis, and the progression from conceptual ideas to practical empirical studies that apply and refine them.

Overall, we find a surprisingly wide and diverse set of techniques and applications in App Store Analysis, highlighting the health and future potential of the field. App Store Analysis opens up an exciting new vista for software engineering research which can connect and deeply understand relationships between social, technical and business facing aspects of software development, deployment and uptake in ways previously impossible due to paucity of data.

\section{ACKNOWLEDGEMENTS}

We thank the anonymous reviewers for their very helpful feedback, and our many colleagues who responded with their valuable comments on an earlier version of this survey. This research was supported by EPRSC (DAASE grant no. EP/J017515).

\section{REFERENCES}

[1] N. A. S. Abdullah, N. I. A. Rusli, and M. F. Ibrahim, "Mobile game size estimation: Cosmic fsm rules, uml mapping model and unity3d game engine," in IEEE Conference on Open Systems (ICOS), 2014, pp. 42-47.

[2] N. Agarwal, R. Karimpour, and G. Ruhe, "Theme-based product release planning: An analytical approach," in System Sciences (HICSS), 2014 47th Hawaii International Conference on. IEEE, 2014, pp. 4739-4748.
[3] A. Al-Subaihin, A. Finkelstein, M. Harman, Y. Jia, W. Martin, F. Sarro, and Y. Zhang, "App store mining and analysis," in Proceedings of the 3rd International Workshop on Software Development Lifecycle for Mobile, DeMobile 2015. ACM, 2015, pp. $1-2$.

[4] A. Al-Subaihin, F. Sarro, S. Black, L. Capra, M. Harman, Y. Jia, and Y. Zhang, "Clustering mobile apps based on mined textual features," in Proceedings of the 10th International Symposium on Empirical Software Engineering and Measurement, ESEM '16. ACM, 2016.

[5] C. Albanesius, "Mobile app reviews: Google 'Bouncer' now scanning Android Market for malware," http://uk.pcmag.com/apps/66697/news/ google-bouncer-now-scanning-android-market-for-mal, 2012.

[6] K. Alharbi and T. Yeh, "Collect, decompile, extract, stats, and diff: Mining design pattern changes in Android apps," in Proceedings of the 17th International Conference on Human-Computer Interaction with Mobile Devices and Services, MobileHCI '15. ACM, 2015, pp. 515-524.

[7] Amazon.com, "Amazon Mechanical Turk," https://www.mturk. com/mturk/welcome, 2013.

[8] androguard, "androguard," https://github.com/androguard/ androguard, 2015.

[9] D. Arp, M. Spreitzenbarth, M. Hübner, H. Gascon, K. Rieck, and C. Siemens, "Drebin: Effective and explainable detection of Android malware in your pocket," in Proceedings of the Annual Symposium on Network and Distributed System Security (NDSS), 2014.

[10] S. Arzt, S. Rasthofer, C. Fritz, E. Bodden, A. Bartel, J. Klein, Y. Le Traon, D. Octeau, and P. McDaniel, "FlowDroid: Precise context, flow, field, object-sensitive and lifecycle-aware taint analysis for Android apps," in Proceedings of the 35th ACM SIGPLAN Conference on Programming Language Design and Implementation (PLDI '14). ACM, 2014, pp. 259-269.

[11] G. Askalidis, "The impact of large scale promotions on the sales and ratings of mobile apps: Evidence from Apple's App Store," CoRR, vol. abs/1506.06857, 2015.

[12] K. W. Y. Au, Y. F. Zhou, Z. Huang, and D. Lie, "Pscout: analyzing the Android permission specification," in Proceedings of the 2012 ACM conference on Computer and communications security. ACM, 2012, pp. 217-228.

[13] V. Avdiienko, K. Kuznetsov, A. Gorla, A. Zeller, S. Arzt, S. Rasthofer, and E. Bodden, "Mining apps for abnormal usage of sensitive data," in Proceedings of the 37th International Conference on Software Engineering - Volume 1, ICSE '15, 2015, pp. 426-436.

[14] N. S. Awang Abu Bakar and I. Mahmud, "Empirical analysis of android apps permissions," in Advanced Computer Science Applications and Technologies (ACSAT), 2013 International Conference on. IEEE, 2013, pp. 406-411.

[15] S. A. Azad, "Empirical studies of Android API usage: Suggesting related API calls and detecting license violations," Master's thesis, Concordia University, 2015.

[16] A. A. Bakar, N. S. Mahmud, and I. Mahmud, "OSSGrab: Software repositories and app store mining tool," Lecture Notes on Software Engineering, vol. 1, no. 3, pp. 219-223, 2013.

[17] D. Barrera, H. G. Kayacik, P. C. van Oorschot, and A. Somayaji, "A methodology for empirical analysis of permission-based security models and its application to Android," in Proceedings of the 17th ACM Conference on Computer and Communications Security, CCS '10. ACM, 2010, pp. 73-84.

[18] A. Bartel, J. Klein, M. Monperrus, and Y. Le Traon, "Static analysis for extracting permission checks of a large scale framework: The challenges and solutions for analyzing Android," Software Engineering, IEEE Transactions on, vol. 40, no. 6, pp. 617-632, 2014.

[19] O. Bastani, S. Anand, and A. Aiken, "Interactively verifying absence of explicit information flows in Android apps," in Proceedings of the 2015 ACM SIGPLAN International Conference on Object-Oriented Programming, Systems, Languages, and Applications, OOPSLA 2015. ACM, 2015, pp. 299-315.

[20] L. Batyuk, M. Herpich, S. A. Camtepe, K. Raddatz, A.-D. Schmidt, and S. Albayrak, "Using static analysis for automatic assessment and mitigation of unwanted and malicious activities within Android applications," in Proceedings of the 2011 6th International Conference on Malicious and Unwanted Software, MALWARE '11. IEEE Computer Society, 2011, pp. 66-72. 
[21] G. Bavota, M. Linares-Vasquez, C. E. Bernal-Cardenas, M. D. Penta, R. Oliveto, and D. Poshyvanyk, "The impact of API changeand fault-proneness on the user ratings of Android apps," IEEE Transactions on Software Engineering, vol. 41, no. 4, pp. 384-407, 2015.

[22] D. L. Ben Lulu and T. Kuflik, "Functionality-based clustering using short textual description: Helping users to find apps installed on their mobile device," in Proceedings of the 2013 International Conference on Intelligent User Interfaces, IUI '13. ACM, 2013, pp. 297-306.

[23] D. L. Ben Lulu and T. Kuflik, "Wise mobile icons organization: Apps taxonomy classification using functionality mining to ease apps finding," Mobile Information Systems, 2015, article ID: 3083450 .

[24] G. Berardi, A. Esuli, T. Fagni, and F. Sebastiani, "Multi-store metadata-based supervised mobile app classification," in Proceedings of the 30th Annual ACM Symposium on Applied Computing, SAC '15. ACM, 2015, pp. 585-588.

[25] P. Bhattacharya, L. Ulanova, I. Neamtiu, and S. C. Koduru, "An empirical analysis of bug reports and bug fixing in open source Android apps," in Proceedings of the 2013 17th European Conference on Software Maintenance and Reengineering, CSMR '13. IEEE Computer Society, 2013, pp. 133-143.

[26] R. Bhoraskar, S. Han, J. Jeon, T. Azim, S. Chen, J. Jung, S. Nath, R. Wang, and D. Wetherall, "Brahmastra: Driving apps to test the security of third-party components," in Proceedings of the 23rd USENIX Conference on Security Symposium, SEC'14. USENIX Association, 2014, pp. 1021-1036.

[27] T. Bläsing, L. Batyuk, A. Schmidt, S. A. Çamtepe, and S. Albayrak, "An Android application sandbox system for suspicious software detection," in Proceedings of the 5th International Conference on Malicious and Unwanted Software, MALWARE'10, 2010, pp. 5562.

[28] M. Böhmer, B. Hecht, J. Schöning, A. Krüger, and G. Bauer, "Falling asleep with angry birds, facebook and kindle: A large scale study on mobile application usage," in Proceedings of the 13th International Conference on Human Computer Interaction with Mobile Devices and Services, MobileHCI '11. ACM, 2011, pp. 47-56.

[29] T. Book, A. Pridgen, and D. S. Wallach, "Longitudinal analysis of android ad library permissions," CoRR, vol. abs/1303.0857, 2013.

[30] H. S. Borges and M. T. Valente, "Mining usage patterns for the Android API," PeerJ Computer Science, no. 1:e12, 2015.

[31] M. Butler, "Android: Changing the mobile landscape," IEEE Pervasive Computing, vol. 10, no. 1, pp. 4-7, 2011.

[32] J. Callaham, "Google says there are now 1.4 billion active Android devices worldwide," http://www.androidcentral.com/ google-says-there-are-now-14-billion-active-android-devices-worl 2015.

[33] B. Carbunar and R. Potharaju, "A longitudinal study of the Google app market," in Proceedings of the 2015 IEEE/ACM International Conference on Advances in Social Networks Analysis and Mining 2015, ASONAM '15. ACM, 2015, pp. 242-249.

[34] L. Cen, C. S. Gates, L. Si, and N. Li, "A probabilistic discriminative model for Android malware detection with decompiled source code," IEEE Trans. Dependable Sec. Comput., vol. 12, no. 4, pp. 400-412, 2015.

[35] L. Cen, D. Kong, H. Jin, and L. Si, "Mobile app security risk assessment: A crowdsourcing ranking approach from user comments," in Proceedings of the 2015 SIAM International Conference on Data Mining, 2015, pp. 658-666.

[36] L. Cen, L. Si, N. Li, and H. Jin, "User comment analysis for Android apps and CSPI detection with comment expansion," in Proceeding of the 1st International Workshop on Privacy-Preserving IR (PIR), 2014, pp. 25-30.

[37] S. Chakradeo, B. Reaves, P. Traynor, and W. Enck, "Mast: Triage for market-scale mobile malware analysis," in Proceedings of the Sixth ACM Conference on Security and Privacy in Wireless and Mobile Networks, WiSec '13. ACM, 2013, pp. 13-24.

[38] R. Chandy and H. Gu, "Identifying spam in the iOS App Store," in Proceedings of the 2Nd Joint WICOW/AIRWeb Workshop on Web Quality, WebQuality '12. ACM, 2012, pp. 56-59.

[39] K. Chen, P. Wang, Y. Lee, X. Wang, N. Zhang, H. Huang, W. Zou, and P. Liu, "Finding unknown malice in 10 seconds: Mass vetting for new threats at the Google-Play scale," in Proceedings of the 24th USENIX Conference on Security Symposium, SEC'15. USENIX Association, 2015, pp. 659-674.
[40] M. Chen and X. Liu, "Predicting popularity of online distributed applications: iTunes App Store case analysis," in Proceedings of the 2011 iConference, iConference '11. ACM, 2011, pp. 661-663.

[41] N. Chen, J. Lin, S. C. H. Hoi, X. Xiao, and B. Zhang, "Ar-miner: Mining informative reviews for developers from mobile app marketplace," in Proceedings of the 36th International Conference on Software Engineering, ICSE '14. ACM, 2014, pp. 767-778.

[42] Y. Chen, H. Xu, Y. Zhou, and S. Zhu, "Is this app safe for children?: A comparison study of maturity ratings on Android and iOS applications," in Proceedings of the 22Nd International Conference on World Wide Web, WWW '13. International World Wide Web Conferences Steering Committee, 2013, pp. 201-212.

[43] P. H. Chia, Y. Yamamoto, and N. Asokan, "Is this app safe?: A large scale study on application permissions and risk signals," in Proceedings of the 21st International Conference on World Wide Web, WWW' '12. ACM, 2012, pp. 311-320.

[44] L. Cocco, K. Mannaro, G. Concas, and M. Marchesi, "Simulation of the best ranking algorithms for an app store," in Mobile Web Information Systems, Lecture Notes in Computer Science. Springer International Publishing, 2014, vol. 8640, pp. 233-247.

[45] S. Comino, F. M. Manenti, and F. Mariuzzo, "Updates management in mobile applications. iTunes vs Google Play," Centre for Competition Policy (CCP), University of East Anglia, 2015.

[46] F. T. Commission, "Complying with COPPA: Frequently Asked Questions," https://www.ftc.gov/tips-advice/business-center/ guidance/complying-coppa-frequently-asked-questions, 2015.

[47] L. Corral and I. Fronza, "Better code for better apps: A study on source code quality and market success of Android applications," in Proceedings of the Second ACM International Conference on Mobile Software Engineering and Systems, MOBILESoft '15. IEEE Press, 2015, pp. 22-32.

[48] COSMIC, "Common software measurement international consortium," http://cosmic-sizing.org/, 2015.

[49] P. Coulton and W. Bamford, "Experimenting through mobile 'apps' and 'app stores'," Int. J. Mob. Hum. Comput. Interact., vol. 3, no. 4, pp. 55-70, 2011.

[50] J. Crussell, C. Gibler, and H. Chen, "Attack of the clones: Detecting cloned applications on Android markets," in Computer SecurityESORICS 2012. Springer, 2012, pp. 37-54.

[51] J. Crussell, C. Gibler, and H. Chen, "Andarwin: Scalable detection of semantically similar Android applications," in Computer Security-ESORICS 2013. Springer, 2013, pp. 182-199.

[52] J. Crussell, R. Stevens, and H. Chen, "Madfraud: Investigating ad fraud in Android applications," in Proceedings of the 12th annual international conference on Mobile systems, applications, and services. ACM, 2014, pp. 123-134.

[53], DAI-Labor, "Androlyzer," https://androlyzer.com/, 2015.

[54] D. Datta and S. Kajanan, "Do app launch times impact their subsequent commercial success? an analytical approach," in Cloud Computing and Big Data (CloudCom-Asia), 2013 International Conference on. IEEE, 2013, pp. 205-210.

[55] L. D'Avanzo, F. Ferrucci, C. Gravino, and P. Salza, "Cosmic functional measurement of mobile applications and code size estimation," in Proceedings of the 30th Annual ACM Symposium on Applied Computing, SAC '15. ACM, 2015, pp. 1631-1636.

[56] Z. Deng, B. Saltaformaggio, X. Zhang, and D. Xu, "iris: Vetting private API abuse in iOS applications," in Proceedings of the 22nd ACM SIGSAC Conference on Computer and Communications Security. ACM, 2015, pp. 44-56.

[57] M. L. Dering and P. McDaniel, "Android Market reconstruction and analysis," in Proceedings of the 2014 IEEE Military Communications Conference, MILCOM '14. IEEE Computer Society, 2014, pp. 300 305.

[58] N. d'Heureuse, F. Huici, M. Arumaithurai, M. Ahmed, K. Papagiannaki, and S. Niccolini, "What's app?: a wide-scale measurement study of smart phone markets," ACM SIGMOBILE Mobile Computing and Communications Review, vol. 16, no. 2, pp. 16-27, 2012.

[59] W. Enck, P. Gilbert, B.-G. Chun, L. P. Cox, J. Jung, P. McDaniel, and A. N. Sheth, "Taintdroid: An information-flow tracking system for realtime privacy monitoring on smartphones," in Proceedings of the 9th USENIX Conference on Operating Systems Design and Implementation, OSDI'10. USENIX Association, 2010, pp. 1-6.

[60] W. Enck, M. Ongtang, and P. McDaniel, "On lightweight mobile phone application certification," in Proceedings of the 16th ACM Conference on Computer and Communications Security, CCS '09. ACM, 2009, pp. 235-245. 
[61] D. Erić, R. Bačík, and I. Fedorko, "Rating decision analysis based on iOS App Store data," Quality Innovation Prosperity, vol. 18, no. 2, pp. 27-37, 2014.

[62] D. Ferreira, V. Kostakos, and A. K. Dey, "Lessons learned from large-scale user studies: Using Android Market as a source of data," Int. J. Mob. Hum. Comput. Interact., vol. 4, no. 3, pp. 28-43, 2012.

[63] F. Ferrucci, C. Gravino, P. Salza, and F. Sarro, "Investigating functional and code size measures for mobile applications," in 41st Euromicro Conference series on Software Engineering and Advanced Applications, SEAA '15. IEEE, 2015, pp. 365-368.

[64] F. Ferrucci, C. Gravino, P. Salza, and F. Sarro, "Investigating functional and code size measures for mobile applications: A replicated study," in 16th International Conference on ProductFocused Software Process Improvement, PROFES '15, 2015.

[65] A. Finkelstein, M. Harman, Y. Jia, W. Martin, F. Sarro, and Y. Zhang, "App store analysis: Mining app stores for relationships between customer, business and technical characteristics," Tech. Rep., 2014, rN/14/10.

[66] R. Francese, C. Gravino, M. Risi, G. Scanniello, and G. Tortora, "On the use of requirements measures to predict software project and product measures in the context of Android mobile apps: a preliminary study," in 41st Euromicro Conference series on Software Engineering and Advanced Applications (SEAA '15). IEEE, 2015, pp. 357-364.

[67] N. Friedman, D. Geiger, and M. Goldszmidt, "Bayesian network classifiers," Mach. Learn., vol. 29, no. 2-3, pp. 131-163, 1997.

[68] B. Fu, J. Lin, L. Li, C. Faloutsos, J. Hong, and N. Sadeh, "Why people hate your app: Making sense of user feedback in a mobile app store," in Proceedings of the 19th ACM SIGKDD International Conference on Knowledge Discovery and Data Mining, KDD '13. ACM, 2013, pp. 1276-1284.

[69] L. V. Galvis Carreño and K. Winbladh, "Analysis of user comments: An approach for software requirements evolution," in Proceedings of the 2013 International Conference on Software Engineering, ICSE '13. IEEE Press, 2013, pp. 582-591.

[70] C. Gao, H. Xu, J. Hu, and Y. Zhou, "Ar-tracker: Track the dynamics of mobile apps via user review mining," in 2015 IEEE Symposium on Service-Oriented System Engineering, SOSE '15, 2015, pp. 284 290.

[71] R. Garg and R. Telang, "Inferring app demand from publicly available data," MIS Q., vol. 37, no. 4, pp. 1253-1264, 2013.

[72] C. Gibler, J. Crussell, J. Erickson, and H. Chen, "AndroidLeaks: Automatically detecting potential privacy leaks in Android applications on a large scale," in Proceedings of the 5th International Conference on Trust and Trustworthy Computing, TRUST'12. Springer-Verlag, 2012, pp. 291-307.

[73] C. Gibler, R. Stevens, J. Crussell, H. Chen, H. Zang, and H. Choi, "Adrob: Examining the landscape and impact of Android application plagiarism," in Proceeding of the 11th annual international conference on Mobile systems, applications, and services. ACM, 2013, pp. 431-444.

[74] GinLemon, "Smart launcher 3 - simple. light. fast." http://www. smartlauncher.net/, 2011.

[75] GitHub, Inc., "Github," https://github.com/, 2014.

[76] W. Glodek and R. Harang, "Rapid permissions-based detection and analysis of mobile malware using random decision forests," in Military Communications Conference, MILCOM 2013-2013 IEEE. IEEE, 2013, pp. 980-985.

[77] M. Gómez, M. Martinez, M. Monperrus, and R. Rouvoy, "When app stores listen to the crowd to fight bugs in the wild," in Proceedings of the 37th International Conference on Software Engineering Volume 2, ICSE '15. IEEE Press, 2015, pp. 567-570.

[78] M. Gomez, R. Rouvoy, M. Monperrus, and L. Seinturier, "A recommender system of buggy app checkers for app store moderators," in 2nd ACM International Conference on Mobile Software Engineering and Systems, D. Dig and Y. Dubinsky, Eds. IEEE, 2015.

[79] A. Gorla, I. Tavecchia, F. Gross, and A. Zeller, "Checking app behavior against app descriptions," in Proceedings of the 2014 International Conference on Software Engineering, ICSE '14. ACM Press, 2014, pp. 292-302.

[80] M. Goul, O. Marjanovic, S. Baxley, and K. Vizecky, "Managing the enterprise business intelligence app store: Sentiment analysis supported requirements engineering," in Proceedings of the 2012 45th Hawaii International Conference on System Sciences, HICSS '12, 2012, pp. 4168-4177.
[81] M. Grace, W. Zhou, X. Jiang, and A.-R. Sadeghi, "Unsafe exposure analysis of mobile in-app advertisements," in Proceedings of the Fifth ACM Conference on Security and Privacy in Wireless and Mobile Networks, WISEC '12. ACM, 2012, pp. 101-112.

[82] M. Grace, Y. Zhou, Q. Zhang, S. Zou, and X. Jiang, "RiskRanker: Scalable and accurate zero-day Android malware detection," in Proceedings of the 10th International Conference on Mobile Systems, Applications, and Services, MobiSys '12. ACM, 2012, pp. 281-294.

[83] X. Gu and S. Kim, "What parts of your apps are loved by users?" in In Proceedings of the 30th IEEE/ACM International Conference on Automated Software Engineering (ASE'15), 2015.

[84] L. Guerrouj, S. Azad, and P. C. Rigby, "The influence of App churn on App success and StackOverflow discussions," in Proceedings of the 22nd International Conference on Software Analysis, Evolution and Reengineering (SANER). IEEE, 2015, pp. 321-330.

[85] J. Gui, S. Mcilroy, M. Nagappan, and W. G. J. Halfond, "Truth in advertising: The hidden cost of mobile ads for software developers," in Proceedings of the 37th International Conference on Software Engineering - Volume 1, ICSE '15. IEEE Press, 2015, pp. 100-110.

[86] E. Guzman, O. Aly, and B. Bruegge, "Retrieving diverse opinions from app reviews," in ACM/IEEE International Symposium on Empirical Software Engineering and Measurement, ESEM '15. IEEE, 2015, pp. 1-10.

[87] E. Guzman, M. El-Haliby, and B. Bruegge, "Ensemble methods for app review classification: An approach for software evolution $(\mathrm{N})$," in 30th IEEE/ACM International Conference on Automated Software Engineering, ASE '15, 2015, pp. 771-776.

[88] E. Guzman and W. Maalej, "How do users like this feature? a fine grained sentiment analysis of app reviews," in 22nd IEEE International Requirements Engineering Conference (RE'14), 2014, pp. $153-162$.

[89] E. Ha and D. Wagner, "Do Android users write about electric sheep? examining consumer reviews in Google Play," in 10th IEEE Consumer Communications and Networking Conference, CCNC '13. IEEE, 2013, pp. 149-157.

[90] Y. J. Ham and H.-W. Lee, "Detection of malicious Android mobile applications based on aggregated system call events," International Journal of Computer and Communication Engineering, vol. 3, no. 2, pp. 149-154, 2014.

[91] S. Hao, B. Liu, S. Nath, W. G. Halfond, and R. Govindan, "PUMA: Programmable UI-Automation for Large-Scale Dynamic Analysis of Mobile Apps," in Proceedings of the 12th International Conference on Mobile Systems, Applications, and Services (MobiSys'14), 2014.

[92] M. Harman, Y. Jia, and Y. Zhang, "App Store Mining and Analysis: MSR for App Stores," in Proceedings of the 9th IEEE Working Conference on Mining Software Repositories, MSR '12, 2012, pp. 108-111.

[93] X. He, W. Dai, G. Cao, R. Tang, M. Yuan, and Q. Yang, "Mining target users for online marketing based on app store data," in 2015 IEEE International Conference on Big Data. IEEE, 2015, pp. 1043-1052.

[94] H. Heitkötter, S. Hanschke, and T. A. Majchrzak, "Evaluating cross-platform development approaches for mobile applications," in Web Information Systems and Technologies - 8th International Conference, WEBIST 2012, Porto, Portugal, April 18-21, 2012, Revised Selected Papers, 2012, pp. 120-138.

[95] N. Henze and S. Boll, "Release your app on Sunday eve: Finding the best time to deploy apps," in Proceedings of the 13th International Conference on Human Computer Interaction with Mobile Devices and Services (MobileHCI'11), 2011, pp. 581-586.

[96] A. Hindle, "Green software engineering: the curse of methodology," in 2016 IEEE 23rd International Conference on Software Analysis, Evolution, and Reengineering (SANER), vol. 5. IEEE, 2016, pp. 46-55.

[97] T.-H. Ho, D. Dean, X. Gu, and W. Enck, "PREC: Practical root exploit containment for Android devices," in Proceedings of the 4th ACM Conference on Data and Application Security and Privacy, CODASPY'14. ACM, 2014, pp. 187-198.

[98] L. Hoon, M. Rodriguez-Garca, R. Vasa, R. Valencia-Garca, and J.-G. Schneider, "App reviews: Breaking the user and developer language barrier," in Trends and Applications in Software Engineering, Advances in Intelligent Systems and Computing. Springer International Publishing, 2016, vol. 405, pp. 223-233.

[99] L. Hoon, R. Vasa, G. Y. Martino, J.-G. Schneider, and K. Mouzakis, "Awesome!: Conveying satisfaction on the app store," in 
Proceedings of the 25th Australian Computer-Human Interaction Conference: Augmentation, Application, Innovation, Collaboration, OzCHI '13. ACM, 2013, pp. 229-232.

[100] L. Hoon, R. Vasa, J.-G. Schneider, and J. Grundy, "An analysis of the mobile app review landscape: trends and implications," Faculty of Information and Communication Technologies, Swinburne University of Technology, Tech. Rep., 2013.

[101] L. Hoon, R. Vasa, J.-G. Schneider, and K. Mouzakis, "A preliminary analysis of vocabulary in mobile app user reviews," in Proceedings of the 24th Australian Computer-Human Interaction Conference, OzCHI '12. ACM, 2012, pp. 245-248.

[102] J. Huang, Z. Li, X. Xiao, Z. Wu, K. Lu, X. Zhang, and G. Jiang, "SUPOR: Precise and scalable sensitive user input detection for Android apps," in Proceedings of the 24th USENIX Conference on Security Symposium, SEC'15. USENIX Association, 2015, pp. 977-992.

[103] W. Huang, Y. Dong, A. Milanova, and J. Dolby, "Scalable and precise taint analysis for Android," in Proceedings of the 2015 International Symposium on Software Testing and Analysis, ISSTA 2015. ACM, 2015, pp. 106-117.

[104] C. Iacob and R. Harrison, "Retrieving and analyzing mobile apps feature requests from online reviews," in Proceedings of the 10th Working Conference on Mining Software Repositories, MSR '13. IEEE Press, 2013, pp. 41-44.

[105] C. Iacob, R. Harrison, and S. Faily, "Online reviews as first class artifacts in mobile app development," in Proceedings of the 5th International Conference on Mobile Computing, Applications, and Services. MobiCASE '13, 2014.

[106] C. Iacob, V. Veerappa, and R. Harrison, "What are you complaining about?: A study of online reviews of mobile applications," in Proceedings of the 27th International BCS Human Computer Interaction Conference, BCS-HCI '13. British Computer Society, 2013, pp. 29:1-29:6.

[107] S.-Y. Ihm, W.-K. Loh, and Y.-H. Park, "App analytic: A study on correlation analysis of app ranking data," International Conference on Cloud and Green Computing (CGC), vol. 0, pp. 561-563, 2013.

[108] J. Jeon, K. K. Micinski, J. A. Vaughan, A. Fogel, N. Reddy, J. S. Foster, and T. Millstein, "Dr. Android and mr. hide: Fine-grained permissions in Android applications," in Proceedings of the Second ACM Workshop on Security and Privacy in Smartphones and Mobile Devices, SPSM '12. ACM, 2012, pp. 3-14.

[109] H. Jiang, H. Ma, Z. Ren, J. Zhang, and X. Li, "What makes a good app description?" in Proceedings of the 6th Asia-Pacific Symposium on Internetware on Internetware, INTERNETWARE 2014. ACM, 2014, pp. 45-53.

[110] Y. Jing, G.-J. Ahn, Z. Zhao, and H. Hu, "Riskmon: Continuous and automated risk assessment of mobile applications," in Proceedings of the 4th ACM Conference on Data and Application Security and Privacy, CODASPY '14. ACM, 2014, pp. 99-110.

[111] Y. Jo and A. H. Oh, "Aspect and sentiment unification model for online review analysis," in Proceedings of the Fourth ACM International Conference on Web Search and Data Mining, WSDM '11. ACM, 2011, pp. 815-824.

[112] M. E. Joorabchi, M. Ali, and A. Mesbah, "Detecting inconsistencies in multi-platform mobile apps," in Proceedings of the 26th IEEE International Symposium on Software Reliability Engineering, ISSRE '15, 2015.

[113] E.-Y. Jung, C. Baek, and J.-D. Lee, "Product survival analysis for the App Store," Marketing Letters, vol. 23, no. 4, pp. 929-941, 2012.

[114] H. Khalid, M. Nagappan, and A. Hassan, "Examining the relationship between FindBugs warnings and end user ratings: A case study on 10,000 Android apps," IEEE Transactions on Software Engineering, 2015.

[115] H. Khalid, "On identifying user complaints of iOS apps," in Proceedings of the 2013 International Conference on Software Engineering, ICSE '13. IEEE Press, 2013, pp. 1474-1476.

[116] H. Khalid, M. Nagappan, E. Shihab, and A. E. Hassan, "Prioritizing the devices to test your app on: A case study of Android game apps," in Proceedings of the 22Nd ACM SIGSOFT International Symposium on Foundations of Software Engineering, FSE 2014. ACM, 2014, pp. 610-620.

[117] H. Khalid, E. Shihab, M. Nagappan, and A. E. Hassan, "What do mobile app users complain about?" IEEE Software, vol. 32, no. 3, pp. 70-77, 2015.

[118] M. Khalid, M. Asif, and U. Shehzaib, "Towards improving the quality of mobile app reviews," International Journal of Information
Technology and Computer Science (IJITCS), vol. 7, no. 10, p. 35, 2015.

[119] M. Khalid, U. Shehzaib, and M. Asif, "A case of mobile app reviews as a crowdsource," International Journal of Information Engineering and Electronic Business (IJIEEB), vol. 7, no. 5, p. 39, 2015.

[120] K. Khanmohammadi, M. R. Rejali, and A. Hamou-Lhadj, "Understanding the service life cycle of Android apps: An exploratory study," in Proceedings of the 5th Annual ACM CCS Workshop on Security and Privacy in Smartphones and Mobile Devices, SPSM '15. ACM, 2015, pp. 81-86.

[121] D. Kim, A. Gokhale, V. Ganapathy, and A. Srivastava, "Detecting plagiarized mobile apps using API birthmarks," Automated Software Engineering, pp. 1-28, 2015.

[122] J. Kim, Y. Park, C. Kim, and H. Lee, "Mobile application service networks: Apple's App Store," Service Business, vol. 8, no. 1, pp. $1-27,2014$.

[123] D. E. Krutz, M. Mirakhorli, S. A. Malachowsky, A. Ruiz, J. Peterson, A. Filipski, and J. Smith, "A dataset of open-source Android applications," in Proceedings of the 12th Working Conference on Mining Software Repositories, MSR '15. IEEE Press, 2015, pp. 522-525.

[124] N. Lageman, M. Lindsey, and W. Glodek, "Detecting malicious Android applications from runtime behavior," in Military Communications Conference, MILCOM 2015-2015 IEEE. IEEE, 2015, pp. 324-329.

[125] G. Lee and T. S. Raghu, "Product portfolio and mobile apps success: Evidence from App Store market," in Proceedings of the 17th Americas Conference on Information Systems AMCIS '11, V. Sambamurthy and M. Tanniru, Eds. Association for Information Systems, 2011.

[126] G. Lee and T. Raghu, "Determinants of mobile apps' success: Evidence from the app store market," Journal of Management Information Systems, vol. 31, no. 2, pp. 133-170, 2014.

[127] L. Li, A. Bartel, T. F. D. A. Bissyande, J. Klein, Y. Le Traon, S. Arzt, S. Rasthofer, E. Bodden, D. Octeau, and P. McDaniel, "Iccta: detecting inter-component privacy leaks in Android apps," in 2015 IEEE/ACM 37th IEEE International Conference on Software Engineering, ICSE '15, 2015.

[128] T.-P. Liang, X. Li, C.-T. Yang, and M. Wang, "What in consumer reviews affects the sales of mobile apps: A multifacet sentiment analysis approach," International Journal of Electronic Commerce, vol. 20, no. 2, pp. 236-260, 2015.

[129] S. Lim, P. Bentley, N. Kanakam, F. Ishikawa, and S. Honiden, "Investigating country differences in mobile app user behavior and challenges for software engineering," IEEE Transactions on Software Engineering (TSE), 2015.

[130] S. L. Lim and P. J. Bentley, "App epidemics: Modelling the effects of publicity in a mobile app ecosystem," in Artificial Life 13: Proceedings of the Thirteenth International Conference on the Simulation and Synthesis of Living Systems (ALIFE), 2012.

[131] S. L. Lim and P. J. Bentley, "How to be a successful app developer: lessons from the simulation of an app ecosystem," ACM SIGEVOlution, vol. 6, no. 1, pp. 2-15, 2012.

[132] S. L. Lim and P. J. Bentley, "Investigating app store ranking algorithms using a simulation of mobile app ecosystems," in Proceedings of the IEEE Congress on Evolutionary Computation, CEC 2013, 2013, pp. 2672-2679.

[133] S. L. Lim, P. J. Bentley, and F. Ishikawa, "The effects of developer dynamics on fitness in an evolutionary ecosystem model of the App Store," IEEE Transactions on Evolutionary Computation (TEVC), vol. PP, 2015.

[134] J. Lin, S. Amini, J. I. Hong, N. Sadeh, J. Lindqvist, and J. Zhang, "Expectation and purpose: Understanding users' mental models of mobile app privacy through crowdsourcing," in Proceedings of the 2012 ACM Conference on Ubiquitous Computing, UbiComp '12. ACM, 2012, pp. 501-510.

[135] J. Lin, K. Sugiyama, M.-Y. Kan, and T.-S. Chua, "Addressing coldstart in app recommendation: Latent user models constructed from twitter followers," in Proceedings of the 36th International ACM SIGIR Conference on Research and Development in Information Retrieval, SIGIR '13. ACM, 2013, pp. 283-292.

[136] J. Lin, K. Sugiyama, M.-Y. Kan, and T.-S. Chua, "New and improved: Modeling versions to improve app recommendation," in Proceedings of the 37th International ACM SIGIR Conference on Research \& Development in Information Retrieval, SIGIR '14. ACM, 2014, pp. 647-656. 
[137] M. Linares-Vásquez, "Supporting evolution and maintenance of Android apps," in Companion Proceedings of the 36th International Conference on Software Engineering, ICSE Companion 2014. ACM, 2014, pp. 714-717.

[138] M. Linares-Vásquez, G. Bavota, C. Bernal-Cárdenas, M. Di Penta, R. Oliveto, and D. Poshyvanyk, "API change and fault proneness: A threat to the success of Android apps," in Proceedings of the 2013 9th Joint Meeting on Foundations of Software Engineering, ESEC/FSE 2013. ACM, 2013, pp. 477-487.

[139] M. Linares-Vásquez, G. Bavota, C. Bernal-Cárdenas, R. Oliveto, M. Di Penta, and D. Poshyvanyk, "Mining energy-greedy API usage patterns in Android apps: An empirical study," in Proceedings of the 11th Working Conference on Mining Software Repositories, MSR '14. ACM, 2014, pp. 2-11.

[140] M. Linares-Vásquez, A. Holtzhauer, C. Bernal-Cárdenas, and D. Poshyvanyk, "Revisiting Android reuse studies in the context of code obfuscation and library usages," in Proceedings of the 11th Working Conference on Mining Software Repositories, MSR '14. ACM, 2014, pp. 242-251.

[141] B. Liu, D. Kong, L. Cen, N. Z. Gong, H. Jin, and H. Xiong, "Personalized mobile app recommendation: Reconciling app functionality and user privacy preference," in Proceedings of the Eighth ACM International Conference on Web Search and Data Mining, WSDM '15. ACM, 2015, pp. 315-324.

[142] B. Liu, B. Liu, H. Jin, and R. Govindan, "Efficient privilege deescalation for ad libraries in mobile apps," in Proceedings of the 13th Annual International Conference on Mobile Systems, Applications, and Services, MobiSys '15. ACM, 2015, pp. 89-103.

[143] B. Liu, S. Nath, R. Govindan, and J. Liu, "Decaf: Detecting and characterizing ad fraud in mobile apps," in Proceedings of the 11th USENIX Conference on Networked Systems Design and Implementation, NSDI'14. USENIX Association, 2014, pp. 5770.

[144] X. Liu and J. Liu, "A two-layered permission-based Android malware detection scheme," in Proceedings of the 2014 2Nd IEEE International Conference on Mobile Cloud Computing, Services, and Engineering, MOBILECLOUD '14. IEEE Computer Society, 2014, pp. $142-148$.

[145] S. Ma, S. Wang, D. Lo, R. H. Deng, and C. Sun, "Active semisupervised approach for checking app behavior against its description," in 39th IEEE Annual Computer Software and Applications Conference, COMPSAC 2015, Taichung, Taiwan, July 1-5, 2015. Volume 2, 2015, pp. 179-184.

[146] W. Maalej and H. Nabil, "Bug report, feature request, or simply praise? on automatically classifying app reviews," Requirements Engineering (RE15), 2015.

[147] I. Malavolta, S. Ruberto, T. Soru, and V. Terragni, "End users' perception of hybrid mobile apps in the Google Play store," in Proceedings of the 4th International Conference on Mobile Services (MS). IEEE, 2015.

[148] I. Malavolta, S. Ruberto, V. Terragni, and T. Soru, "Hybrid mobile apps in the Google Play store: an exploratory investigation," in Proceedings of the 2nd ACM International Conference on Mobile Software Engineering and Systems. ACM, 2015.

[149] L. M. Manevitz and M. Yousef, "One-class svms for document classification," J. Mach. Learn. Res., vol. 2, pp. 139-154, 2002.

[150] K. Mao, L. Capra, M. Harman, and Y. Jia, "A survey of the use of crowdsourcing in software engineering," Tech. Rep., 2016, $\mathrm{rN} / 15 / 01$.

[151] K. Mao, M. Harman, and Y. Jia, "Sapienz: multi-objective automated testing for android applications," in Proceedings of the 25th International Symposium on Software Testing and Analysis. ACM, 2016, pp. 94-105.

[152] marketsandmarkets.com, "World Mobile Applications Market - Advanced Technologies, Global Forecast," http://www.marketsandmarkets.com/Market-Reports/ mobile-applications-228.html, 2010.

[153] W. Martin, "Causal impact for app store analysis," in Companion Proceedings of the 38th International Conference on Software Engineering, ICSE Companion '16. ACM, 2016.

[154] W. Martin, M. Harman, Y. Jia, F. Sarro, and Y. Zhang, "The app sampling problem for app store mining," in Proceedings of the 12th IEEE Working Conference on Mining Software Repositories, MSR '15, 2015, pp. 123-133.

[155] W. Martin, F. Sarro, and M. Harman, "Causal impact analysis applied to app releases in Google Play and Windows Phone Store," University College London, Tech. Rep., 2015, rN/15/07.
[156] W. Martin, F. Sarro, and M. Harman, "Causal impact analysis for app releases in Google Play," in Proceedings of the 2016 24th ACM SIGSOFT International Symposium on the Foundations of Software Engineering, FSE '16, 2016, to appear.

[157] W. Martin, F. Sarro, Y. Jia, Y. Zhang, and M. Harman, "A survey of app store analysis for software engineering," University College London, Tech. Rep., 2016, rN/16/02.

[158] T. McDonnell, B. Ray, and M. Kim, "An empirical study of API stability and adoption in the Android ecosystem," in Proceedings of the 2013 IEEE International Conference on Software Maintenance, ICSM '13. IEEE Computer Society, 2013, pp. 70-79.

[159] S. Mcllroy, N. Ali, and A. E. Hassan, "Fresh apps: an empirical study of frequently-updated mobile apps in the google play store," Empirical Software Engineering, pp. 1-25, 2015.

[160] S. McIlroy, N. Ali, H. Khalid, and A. E. Hassan, "Analyzing and automatically labelling the types of user issues that are raised in mobile app reviews," Empirical Software Engineering, pp. 1-40, 2015.

[161] S. McIlroy, W. Shang, N. Ali, and A. Hassan, "Is it worth responding to reviews? a case study of the top free apps in the Google Play store," IEEE Software, vol. PP, 2015.

[162] D. Mimno, H. Wallach, E. Talley, M. Leenders, and A. McCallum, "Optimizing semantic coherence in topic models," in Proceedings of the 2011 Conference on Empirical Methods in Natural Language Processing. Association for Computational Linguistics, 2011, pp. 262-272.

[163] R. Minelli and M. Lanza, "Samoa - a visual software analytics platform for mobile applications," in Proceedings of ICSM 2013 (29th International Conference on Software Maintenance). IEEE CS Press, 2013, pp. 476-479.

[164] R. Minelli and M. Lanza, "Software analytics for mobile applications-insights \&amp; lessons learned," 2013 15th European Conference on Software Maintenance and Reengineering, vol. 0, pp. 144-153, 2013.

[165] I. J. Mojica, M. Nagappan, B. Adams, T. Berger, S. Dienst, and A. E. Hassan, "An examination of the current rating system used in mobile app stores," IEEE Software, vol. PP, 2015.

[166] S. Mokarizadeh, M. T. Rahman, and M. Matskin, "Mining and analysis of apps in Google Play," in Web Information Systems and Technologies - 9th International Conference, WEBIST '13, 2013.

[167] J. E. Montandon, H. Borges, D. Felix, and M. T. Valente, "Documenting APIs with examples: Lessons learned with the APIMiner platform," in 20th Working Conference on Reverse Engineering (WCRE). IEEE, 2013, pp. 401-408.

[168] K. Moran, M. Linares-Vásquez, C. Bernal-Cárdenas, and D. Poshyvanyk, "Auto-completing bug reports for Android applications," in Proceedings of the 2015 10th Joint Meeting on Foundations of Software Engineering. ACM, 2015, pp. 673-686.

[169] P. Mutchler, A. Doupé, J. Mitchell, C. Kruegel, and G. Vigna, "A Large-Scale Study of Mobile Web App Security," in Proceedings of the Mobile Security Technologies Workshop (MoST), 2015.

[170] A. Mller, S. Diewald, L. Roalter, T. U. Mnchen, F. Michahelles, and M. Kranz, "Update behavior in app markets and security implications: A case study in Google Play," in In Proc. of the 3rd Intl. Workshop on Research in the Large. Held in Conjunction with Mobile HCI, 2012, pp. 3-6.

[171] M. Nagappan and E. Shihab, "Future trends in software engineering research for mobile apps," in 23rd IEEE International Conference on Software Analysis, Evolution, and Reengineering SANER '16, 2016.

[172] M. Nayebi and G. Ruhe, "Trade-off service portfolio planning-a case study on mining the Android app market," PeerJ PrePrints, 2015.

[173] Y. Y. Ng, H. Zhou, Z. Ji, H. Luo, and Y. Dong, "Which Android app store can be trusted in China?" in Proceedings of the 2014 IEEE 38th Annual Computer Software and Applications Conference, COMPSAC '14. IEEE Computer Society, 2014, pp. 509-518.

[174] J. Oh, D. Kim, U. Lee, J.-G. Lee, and J. Song, "Facilitating developer-user interactions with mobile app review digests," in CHI '13 Extended Abstracts on Human Factors in Computing Systems, CHI EA '13. ACM, 2013, pp. 1809-1814.

[175] D. Pagano and W. Maalej, "User feedback in the appstore: An empirical study." in Proceedings of the 21st. IEEE International Requirements Engineering Conference (RE) '13. IEEE, 2013.

[176] F. Palomba, M. Linares-Vásquez, G. Bavota, R. Oliveto, M. Di Penta, D. Poshyvanyk, and A. De Lucia, "User reviews matter! tracking crowdsourced reviews to support evolution of 
successful apps," in 31st International Conference on Software Maintenance and Evolution, ICSME '15, 2015.

[177] R. Pandita, X. Xiao, W. Yang, W. Enck, and T. Xie, "Whyper: Towards automating risk assessment of mobile applications," in Proceedings of the 22Nd USENIX Conference on Security, SEC'13. USENIX Association, 2013, pp. 527-542.

[178] S. Panichella, A. D. Sorbo, E. Guzman, A. Visaggio, G. Canfora, and H. Gall, "How can i improve my app? classifying user reviews for software maintenance and evolution," 31st IEEE International Conference on Software Maintenance and Evolution, 2015.

[179] D. H. Park, M. Liu, C. Zhai, and H. Wang, "Leveraging user reviews to improve accuracy for mobile app retrieval," in Proceedings of the 38th International ACM SIGIR Conference on Research and Development in Information Retrieval, SIGIR '15. ACM, 2015, pp. 533-542.

[180] N. Peiravian and X. Zhu, "Machine learning for Android malware detection using permission and API calls," in Proceedings of the 2013 IEEE 25th International Conference on Tools with Artificial Intelligence, ICTAI '13. IEEE Computer Society, 2013, pp. 300305.

[181] H. Peng, C. S. Gates, B. P. Sarma, N. Li, Y. Qi, R. Potharaju, C. NitaRotaru, and I. Molloy, "Using probabilistic generative models for ranking risks of Android apps," in ACM Conference on Computer and Communications Security. ACM, 2012, pp. 241-252.

[182] T. Petsas, A. Papadogiannakis, M. Polychronakis, E. P. Markatos, and T. Karagiannis, "Rise of the planet of the apps: A systematic study of the mobile app ecosystem," in Proceedings of the 2013 Conference on Internet Measurement Conference, IMC '13. ACM, 2013, pp. 277-290.

[183] R. Potharaju, A. Newell, C. Nita-Rotaru, and X. Zhang, "Plagiarizing smartphone applications: Attack strategies and defense techniques," in Proceedings of the 4th International Conference on Engineering Secure Software and Systems (ESSoS'12). SpringerVerlag, 2012, pp. 106-120.

[184] T. Preuss, "Mobile applications, function points and cost estimating," in International Cost Estimation \& Analysis Association Conference, 2013.

[185] T. Preuss, "Mobile applications, functional analysis, and the customer experience," in The IFPUG Guide to IT and Software Measurement, IFPUG, Ed. Auerbach Publications, 2012, pp. 408433.

[186] Z. Qu, V. Rastogi, X. Zhang, Y. Chen, T. Zhu, and Z. Chen, "AutoCog: Measuring the description-to-permission fidelity in Android applications," in Proceedings of the 2014 ACM SIGSAC Conference on Computer and Communications Security. ACM, 2014, pp. 1354-1365.

[187] V. Rastogi, Y. Chen, and W. Enck, "Appsplayground: automatic security analysis of smartphone applications," in Proceedings of the third ACM conference on Data and application security and privacy. ACM, 2013, pp. 209-220.

[188] L. Ravindranath, S. Nath, J. Padhye, and H. Balakrishnan, "Automatic and scalable fault detection for mobile applications," in Proceedings of the 12th Annual International Conference on Mobile Systems, Applications, and Services, MobiSys '14. ACM, 2014, pp. 190-203.

[189] A.-D. Rein and J. Münch, "Feature prioritization based on mockpurchase: A mobile case study," in Proceedings of the Lean Enterprise Software and Systems Conference (LESS 2013). Springer, 2013, pp. 165-179.

[190] S. Robertson, "Understanding inverse document frequency: On theoretical arguments for idf," Journal of Documentation, vol. 60, 2004.

[191] S. Roy, J. DeLoach, Y. Li, N. Herndon, D. Caragea, X. Ou, V. P. Ranganath, H. Li, and N. Guevara, "Experimental study with real-world data for Android app security analysis using machine learning," in Proceedings of the 31st Annual Computer Security Applications Conference, ACSAC 2015. ACM, 2015, pp. 81-90.

[192] I. J. M. Ruiz, B. Adams, M. Nagappan, S. Dienst, T. Berger, and A. E. Hassan, "A large scale empirical study on software reuse in mobile apps," IEEE Software, vol. 31, no. 2, pp. 78-86, 2014.

[193] I. J. M. Ruiz, M. Nagappan, B. Adams, T. Berger, S. Dienst, and A. E. Hassan, "Impact of ad libraries on ratings of Android mobile apps," IEEE Software, vol. 31, no. 6, pp. 86-92, 2014.

[194] I. J. M. Ruiz, M. Nagappan, B. Adams, T. Berger, S. Dienst, and A. E. Hassan, "Analyzing ad library updates in android apps," IEEE Software, vol. 33, no. 2, pp. 74-80, 2016.
[195] I. J. M. Ruiz, M. Nagappan, B. Adams, and A. E. Hassan, "Understanding reuse in the Android market," in 20th IEEE International Conference on Program Comprehension, ICPC '12, 2012, pp. 113122.

[196] A. Sahami Shirazi, N. Henze, A. Schmidt, R. Goldberg, B. Schmidt, and H. Schmauder, "Insights into layout patterns of mobile user interfaces by an automatic analysis of Android apps," in Proceedings of the 5th ACM SIGCHI Symposium on Engineering Interactive Computing Systems, EICS '13. ACM, 2013, pp. 275-284.

[197] B. Sanz, I. Santos, C. Laorden, X. Ugarte-Pedrero, and P. G. Bringas, "On the automatic categorisation of Android applications," in CCNC'12, 2012, pp. 149-153.

[198] B. Sanz, I. Santos, C. Laorden, X. Ugarte-Pedrero, J. Nieves, P. G. Bringas, and G. Álvarez, "MAMA: Manifest analysis for malware detection in Android," Cybernetics and Systems - Intelligent Network Security and Survivability, vol. 44, no. 6-7, pp. 469-488, 2013.

[199] B. Sanz, I. Santos, J. Nieves, C. Laorden, I. Alonso-Gonzalez, and P. G. Bringas, "Mads: malicious Android applications detection through string analysis," in Network and System Security. Springer, 2013, pp. 178-191.

[200] B. Sanz, I. Santos, X. Ugarte-Pedrero, C. Laorden, J. Nieves, and P. G. Bringas, "Instance-based anomaly method for Android malware detection," in Proceedings of the 10th International Conference on Security and Cryptography, SECRYPT '13, 2013, pp. 387-394.

[201] F. Sarro, "The UCLAppA repository: A repository of research articles on mobile software engineering and app store analysis," http://www0.cs.ucl.ac.uk/staff/F.Sarro/projects/UCLappA/ UCLappArepository.html.

[202] F. Sarro, A. A. Al-Subaihin, M. Harman, Y. Jia, W. Martin, and Y. Zhang, "Feature lifecycles as they spread, migrate, remain and die in app stores," in Proceedings of the Requirements Engineering Conference, 23rd IEEE International (RE'15). IEEE, 2015.

[203] J. Schütte, R. Fedler, and D. Titze, "ConDroid: Targeted dynamic analysis of Android applications," in 29th IEEE International Conference on Advanced Information Networking and Applications, AINA '15, 2015, pp. 571-578.

[204] S. Seneviratne, H. Kolamunna, and A. Seneviratne, "A measurement study of tracking in paid mobile applications," in Proceedings of the 8th ACM Conference on Security \& Privacy in Wireless and Mobile Networks, WiSec '15. ACM, 2015, pp. 7:1-7:6.

[205] S. Seneviratne, A. Seneviratne, M. A. Kaafar, A. Mahanti, and P. Mohapatra, "Early detection of spam mobile apps," in Proceedings of the 24th International Conference on World Wide Web, WWW' 15 . International World Wide Web Conferences Steering Committee, 2015, pp. 949-959.

[206] G. Sethumadhavan, "Sizing Android mobile applications," 2011, presentation at 6th IFPUG International Software Measurement and Analysis Conference, ISMA ' 11.

[207] A. Shabtai, Y. Fledel, and Y. Elovici, "Automated static code analysis for classifying Android applications using machine learning," in Proceedings of the 2010 International Conference on Computational Intelligence and Security, CIS '10. IEEE Computer Society, 2010, pp. 329-333.

[208] C. Sharma, "Sizing up the global mobile apps market," Report, Chetan Sharma Consulting, Issaquah, WA, 2010.

[209] K. Shi and K. Ali, "Getjar mobile application recommendations with very sparse datasets," in Proceedings of the 18th ACM SIGKDD international conference on Knowledge discovery and data mining. ACM, 2012, pp. 204-212.

[210] C. Shuler, "iLearnII; an analysis of the education category of the iTunes App Store," The Joan Ganz Cooney Center at Sesame Workshop, 2012.

[211] R. Stevens, J. Ganz, V. Filkov, P. Devanbu, and H. Chen, "Asking for (and about) permissions used by Android apps," in Proceedings of the 10th Working Conference on Mining Software Repositories, MSR '13. IEEE Press, 2013, pp. 31-40.

[212] Z. Svedic, "The effect of informational signals on mobile apps sales ranks across the globe," Ph.D. dissertation, SIMON FRASER UNIVERSITY, 2015.

[213] M. D. Syer, B. Adams, Y. Zou, and A. E. Hassan, "Exploring the development of micro-apps: A case study on the BlackBerry and Android platforms," in Proceedings of the 2011 IEEE 11th International Working Conference on Source Code Analysis and Manipulation, SCAM '11. IEEE Computer Society, 2011, pp. 5564. 
[214] M. D. Syer, M. Nagappan, B. Adams, and A. E. Hassan, "Studying the relationship between source code quality and mobile platform dependence," Software Quality Journal, vol. 23, no. 3, pp. 485508, 2015.

[215] M. D. Syer, M. Nagappan, A. E. Hassan, and B. Adams, "Revisiting prior empirical findings for mobile apps: An empirical case study on the 15 most popular open-source Android apps," in Proceedings of the 2013 Conference of the Center for Advanced Studies on Collaborative Research, CASCON '13. IBM Corp., 2013, pp. 283297.

[216] J. Tan, K. Nguyen, M. Theodorides, H. Negrón-Arroyo, C. Thompson, S. Egelman, and D. Wagner, "The effect of developer-specified explanations for permission requests on smartphone user behavior," in Proceedings of the SIGCHI Conference on Human Factors in Computing Systems. ACM, 2014, pp. 91-100.

[217] P. Teufl, M. Ferk, A. Fitzek, D. Hein, S. Kraxberger, and C. Orthacker, "Malware detection by applying knowledge discovery processes to application metadata on the Android Market (Google Play)," Security and Communication Networks, 2013.

[218] P. Teufl, S. Kraxberger, C. Orthacker, G. Lackner, M. Gissing, A. Marsalek, J. Leibetseder, and O. Prevenhueber, "Android Market analysis with activation patterns," in Security and Privacy in Mobile Information and Communication Systems (MobiSec), Lecture Notes of the Institute for Computer Sciences, Social Informatics and Telecommunications Engineering. Springer Berlin Heidelberg, 2012, vol. 94, pp. 1-12.

[219] Y. Tian, M. Nagappan, D. Lo, and A. E. Hassan, "What are the characteristics of high-rated apps? a case study on free Android applications," in 31st International Conference on Software Maintenance and Evolution, ICSME '15, 2015, pp. 1-10.

[220] Y.-X. Tong, J. She, and L. Chen, "Towards better understanding of app functions," Journal of Computer Science and Technology, vol. 30, no. 5, pp. 1130-1140, 2015.

[221] Université du Luxembourg, "Androzoo," https://androzoo.uni. lu/, 2016.

[222] S. Vakulenko, O. Müller, and J. v. Brocke, "Enriching iTunes App Store categories via topic modeling," in International Conference on Information Systems (ICIS'14), 2014.

[223] H. van Heeringen and E. Van Gorp, "Measure the functional size of a mobile app: Using the cosmic functional size measurement method," in Software Measurement and the International Conference on Software Process and Product Measurement (IWSMMENSURA), 2014 Joint Conference of the International Workshop on. IEEE, 2014, pp. 11-16.

[224] R. Vasa, L. Hoon, K. Mouzakis, and A. Noguchi, "A preliminary analysis of mobile app user reviews," in Proceedings of the 24th Australian Computer-Human Interaction Conference, OzCHI '12. ACM, 2012, pp. 241-244.

[225] N. Viennot, "GitHub - nviennot/playdrone: Google Play Crawler," https://github.com/nviennot/playdrone, 2014.

[226] N. Viennot, E. Garcia, and J. Nieh, "A measurement study of Google Play," in The 2014 ACM international conference on Measurement and modeling of computer systems, SIGMETRICS '14. ACM, 2014, pp. 221-233.

[227] L. Vigneri, J. Chandrashekar, I. Pefkianakis, and O. Heen, "Taming the Android appstore: Lightweight characterization of Android applications," CoRR, vol. abs/1504.06093, 2015.

[228] L. Villarroel Pérez, "Mining mobile apps reviews to support release planning," Master's thesis, ETSI Informatica, 2015.

[229] VirusShare, "Virusshare.com," http://virusshare.com/, 2011.

[230] Vision Mobile, "Developer Economics 2013: The tools report," http://www.visionmobile.com/product/ developer-economics-2013-the-tools-report/, 2013.

[231] Vision Mobile, "Developer Economics Q1 2015: State of the Developer Nation," http://www.visionmobile.com/product/ developer-economics-q1-2015-state-developer-nation/, 2015.

[232] A. von Rhein, T. Berger, N. S. Johansson, M. M. Hardø, and S. Apel, "Lifting inter-app data-flow analysis to large app sets," Fakultät für Informatik und Mathematik, Universität Passau, Tech. Rep., 2015.

[233] P. M. Vu, T. T. Nguyen, H. V. Pham, and T. T. Nguyen, "Mining user opinions in mobile app reviews: A keyword-based approach," in Proceedings of the 30th IEEE/ACM International Conference on Automated Software Engineering (ASE '15). IEEE, 2015, pp. 749759.

[234] P. M. Vu, H. V. Pham, T. T. Nguyen, and T. T. Nguyen, "Tool support for analyzing mobile app reviews," in Proceedings of the 30th IEEE/ACM International Conference on Automated Software Engineering (ASE'15). IEEE, 2015, pp. 789-794.

[235] T. J. Walid Maalej, Maleknaz Nayebi and G. Ruhe, "Toward datadriven requirements engineering," IEEE Software Jan/Feb 2016: Special Issue on the Future of Software Engineering, 2016, to appear.

[236] M. Wan, Y. Jin, D. Li, and W. G. Halfond, "Detecting display energy hotspots in Android apps," in Software Testing, Verification and Validation (ICST), 2015 IEEE 8th International Conference on. IEEE, 2015, pp. 1-10.

[237] H. Wang, Y. Guo, Z. Ma, and X. Chen, "WuKong: A scalable and accurate two-phase approach to Android app clone detection," in Proceedings of the 2015 International Symposium on Software Testing and Analysis, ISSTA 2015. ACM, 2015, pp. 71-82.

[238] H. Wang, J. Hong, and Y. Guo, "Using text mining to infer the purpose of permission use in mobile apps," in Proceedings of the 2015 ACM International Joint Conference on Pervasive and Ubiquitous Computing, UbiComp '15. ACM, 2015, pp. 11071118.

[239] W. Wang, X. Wang, D. Feng, J. Liu, Z. Han, and X. Zhang, "Exploring permission-induced risk in Android applications for malicious application detection," IEEE Transactions on Information Forensics and Security, pp. 1869-1882, 2014.

[240] Y. Wang, J. Zheng, C. Sun, and S. Mukkamala, "Quantitative security risk assessment of Android permissions and applications," in Proceedings of the 27th International Conference on Data and Applications Security and Privacy XXVII, DBSec'13. SpringerVerlag, 2013, pp. 226-241.

[241] M. Wano and J. Iio, "Relationship between reviews at app store and the categories for software," in Proceedings of the 2014 17th International Conference on Network-Based Information Systems, NBIS '14. IEEE Computer Society, 2014, pp. 580-583.

[242] T. Watanabe, M. Akiyama, T. Sakai, H. Washizaki, and T. Mori, "Understanding the inconsistencies between text descriptions and the use of privacy-sensitive resources of mobile apps," in Eleventh Symposium On Usable Privacy and Security (SOUPS 2015). USENIX Association, 2015.

[243] S. Wenxuan and Y. Airu, "Interoperability-enriched app recommendation," in 2014 IEEE International Conference on Data Mining Workshop (ICDMW). IEEE, 2014, pp. 1242-1245.

[244] C. Wohlin, "Guidelines for snowballing in systematic literature studies and a replication in software engineering," in Proceedings of the 18th International Conference on Evaluation and Assessment in Software Engineering, EASE '14. ACM, 2014, pp. 38:1-38:10.

[245] Z. Xie and S. Zhu, "AppWatcher: Unveiling the underground market of trading mobile app reviews," in Proceedings of the 8th ACM Conference on Security \& Privacy in Wireless and Mobile Networks, WiSec '15. ACM, 2015, pp. 10:1-10:11.

[246] W. Xu, F. Zhang, and S. Zhu, "Permlyzer: Analyzing permission usage in Android applications," in 24th IEEE International Symposium on Software Reliability Engineering, ISSRE '13. IEEE, 2013, pp. 400-410.

[247] W. Yang, X. Xiao, B. Andow, S. Li, T. Xie, and W. Enck, "AppContext: Differentiating malicious and benign mobile app behaviors using context," in Proceedings of the 37th International Conference on Software Engineering - Volume 1, ICSE '15, 2015, pp. 303-313.

[248] Y. Yang, J. Stella Sun, and M. W. Berry, "APPIC: Finding the hidden scene behind description files for Android apps," Dept. of Electrical Engineering and Computer Science University of Tennessee, Tech. Rep., 2014.

[249] P. Yin, P. Luo, W.-C. Lee, and M. Wang, "App recommendation: A contest between satisfaction and temptation," in Proceedings of the Sixth ACM International Conference on Web Search and Data Mining, WSDM '13. ACM, 2013, pp. 395-404.

[250] F. Zhang, H. Huang, S. Zhu, D. Wu, and P. Liu, "Viewdroid: Towards obfuscation-resilient mobile application repackaging detection," in Proceedings of the 7th ACM Conference on Security and Privacy in Wireless \& Mobile Networks, WiSec '14, 2014, pp. 2536.

[251] M. Zhang, Y. Duan, Q. Feng, and H. Yin, "Towards automatic generation of security-centric descriptions for Android apps," in Proceedings of the 22Nd ACM SIGSAC Conference on Computer and Communications Security, CCS '15. ACM, 2015, pp. 518-529.

[252] N. Zhong and F. Michahelles, "Google Play is not a long tail market: An empirical analysis of app adoption on the Google Play app market," in Proceedings of the 28th Annual ACM Symposium on Applied Computing, SAC '13. ACM, 2013, pp. 499-504. 
[253] Y. Zhou and X. Jiang, "Dissecting Android malware: Characterization and evolution," in Security and Privacy (SP), 2012 IEEE Symposium on. IEEE, 2012, pp. 95-109.

[254] Y. Zhou, L. Wu, Z. Wang, and X. Jiang, "Harvesting developer credentials in Android apps," in Proceedings of the 8th ACM Conference on Security \& Privacy in Wireless and Mobile Networks, WiSec '15. ACM, 2015, pp. 23:1-23:12.

[255] H. Zhu, C. Liu, Y. Ge, H. Xiong, and E. Chen, "Popularity modeling for mobile apps: A sequential approach," IEEE Transactions on Cybernetics, vol. 45, no. 7, pp. 1303-1314, 2014.

[256] H. Zhu, H. Cao, E. Chen, H. Xiong, and J. Tian, "Exploiting enriched contextual information for mobile app classification," in Proceedings of the 21st ACM international conference on Information and knowledge management. ACM, 2012, pp. 1617-1621.

[257] H. Zhu, E. Chen, H. Xiong, H. Cao, and J. Tian, "Mobile app classification with enriched contextual information," IEEE Transactions on Mobile Computing, vol. 13, no. 7, pp. 1550-1563, 2014.

[258] H. Zhu, E. Chen, H. Xiong, K. Yu, H. Cao, and J. Tian, "Mining mobile user preferences for personalized context-aware recommendation," ACM Transactions on Intelligent Systems and Technology, vol. 5, no. 4, p. 58, 2015.

[259] H. Zhu, H. Xiong, Y. Ge, and E. Chen, "Ranking fraud detection for mobile apps: A holistic view," in Proceedings of the 22Nd ACM International Conference on Conference on Information \& Knowledge Management, CIKM '13. ACM, 2013, pp. 619-628.

[260] H. Zhu, H. Xiong, Y. Ge, and E. Chen, "Mobile app recommendations with security and privacy awareness," in Proceedings of the 20th ACM SIGKDD International Conference on Knowledge Discovery and Data Mining, KDD '14. ACM, 2014, pp. 951-960.

[261] H. Zhu, H. Xiong, Y. Ge, and E. Chen, "Discovery of ranking fraud for mobile apps," Knowledge and Data Engineering, IEEE Transactions on, vol. 27, no. 1, pp. 74-87, 2015.

[262] J. Zhu, Z. Guan, Y. Yang, L. Yu, H. Sun, and Z. Chen, "Permissionbased abnormal application detection for Android," in Proceedings of the 14th International Conference on Information and Communications Security, ICICS'12. Springer-Verlag, 2012, pp. 228-239.

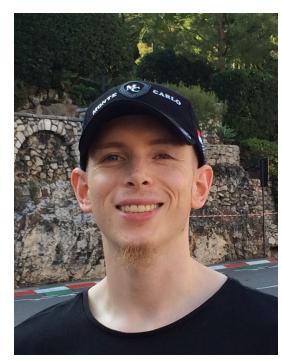

William Martin is a $\mathrm{PhD}$ candidate in computer science at University College London, where he is part of the CREST centre and the UCLappA research group. He is supervised by Mark Harman, Yue Jia and Federica Sarro. His research interests include temporal and causal analyses, machine learning and of course app store analysis. He is a member of Appredict, an app store analytics company that has spun out from UCLappA.

Federica Sarro is a Senior Research

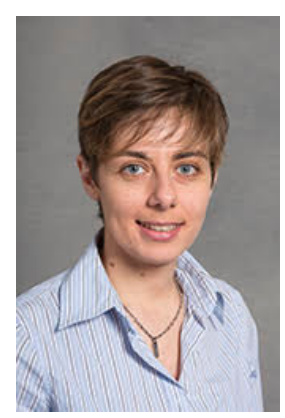

Associate in the CREST centre, Department of Computer Science, at University College London. She is currently member of the UCLs App Store Analysis Group (UCLappA) and of Appredict, an app store analytics company spun out from the UCLappA group. Her main research areas are Empirical and Search Based Software Engineering, with a particular interest in predictive modelling for cost and quality estimations, and app stores mining and analysis. She has published over 50 papers and served as program committee member of more than 40 international events in her area of expertise; in 2015 she has been elected in the steering committee of SSBSE. She has also been program co-chair of SSBSE 2016 and GECCO 2017, co-organiser of WAMA 2016, and track/publicity chair for other international events.

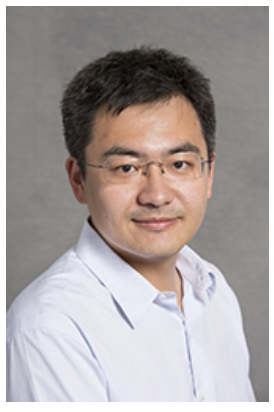

Yue Jia is a lecturer in the Department of Computer Science at University College London. He is currently leading the App Store Analysis Group (UCLappA), which analyses mobile app store ecosystems to understand their key mechanisms. His research interests cover app store analysis, software testing and search-based software engineering. Dr. Jia is also co-director of Appredict, an app store analytics company, spun out from UCL's UCLappA group, and director of MaJiCKe, an automated test data generation start up.

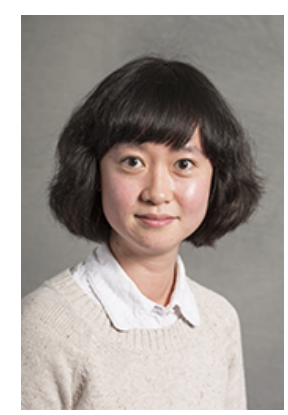

Yuanyuan Zhang is currently a Principal research associate in the CREST centre, University College London. She received her $\mathrm{PhD}$ in Software Engineering from Kings College London in 2010. Her research interests include searchbased requirements optimisation, app store mining and analysis and evolutionary computation. She has published over 20 papers including TSE, RE and $\mathrm{RE}$ journal. She is the co-author of several invited keynote papers at leading international conferences, including SPLC 2014 and ICST 2015. She has served on program committees including REFSQ, GECCO, SSBSE, AIRE, MOBS, RELENG, RET, review committee for RE and as the program co-chair for SSBSE 2013 and been elected onto the steering committee (2013-2016) for SSBSE.

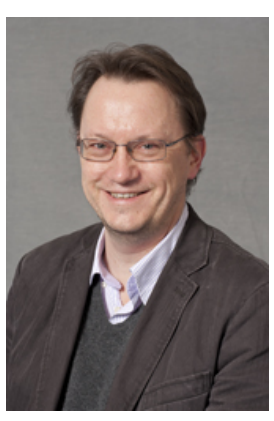

Mark Harman is professor of Software Engineering in the Department of Computer Science at University College London, where he directs the CREST centre and is Head of Software Systems Engineering. He is known for work on source code analysis, software testing, app store analysis and Search Based Software Engineering (SBSE), a field he co-founded and which has grown rapidly to include over 1,600 authors spread over more than 40 countries. His work has been used by many organisations including Daimler, Ericsson, Google, Huawei, Microsoft and Visa. Prof. Harman is co-director of Appredict, an app store analytics company, spun out from UCL's UCLappA group, and chief scientific advisor to MaJiCKe, and automated test data generation start up. 